\title{
HeCS-red: Dense Hectospec Surveys of redMaPPer-selected Clusters
}

\author{
Kenneth J. Rines ${ }^{1}$, Margaret J. Geller ${ }^{2}$ (1) , Antonaldo Diaferio ${ }^{3,4}$, Ho Seong Hwang ${ }^{5}$ (1) , and Jubee Sohn $^{2}$ (1) \\ ${ }^{1}$ Department of Physics \& Astronomy, Western Washington University, Bellingham, WA 98225, USA; kenneth.rines@wwu.edu \\ ${ }^{2}$ Smithsonian Astrophysical Observatory, 60 Garden Street, Cambridge, MA 02138, USA \\ ${ }^{3}$ Universita' di Torino, Dipartimento di Fisica, Torino, Italy \\ ${ }^{4}$ Istituto Nazionale di Fisica Nucleare (INFN), Sezione di Torino, Torino, Italy \\ ${ }^{5}$ Quantum Universe Center, Korea Institute for Advanced Study, 85 Hoegiro, Dongdaemun-gu, Seoul 02455, Republic of Korea \\ Received 2017 November 30; revised 2018 May 19; accepted 2018 June 4; published 2018 August 3
}

\begin{abstract}
We use dense redshift surveys to explore the properties of galaxy clusters selected from the redMaPPer catalog. Our new survey, Hectospec Cluster Survey of red-sequence-selected clusters (HeCS-red), includes 10,589 new or remeasured redshifts from MMT/Hectospec observations of 27 redMaPPer clusters at redshifts $z=0.08-0.25$ with large estimated richnesses (richness estimate $\lambda>64$ ). Our spectra confirm that each candidate cluster corresponds to a large overdensity in redshift space. The redMaPPer photometric redshifts have a slight bias toward higher redshifts. We measure the relation between velocity dispersion $\sigma_{p}$ and $\lambda$. The relation shows intrinsic scatter of $24 \%$ (and a range of $2 \mathrm{x}$ ) in velocity dispersion at fixed richness. We extend our analysis to HeCS-red-ext, a sample that includes several clusters selected by X-ray flux or Sunyaev-Zeldovich signal. The heterogeneous sample of 121 clusters in HeCS-red-ext shows similar intrinsic scatter in the $\sigma_{p}-\lambda$ relation as the HeCS-red sample, but the range of $\sigma_{p}$ at fixed richness increases to $3 \mathrm{x}$. We evaluate the membership probability estimates $P_{\text {mem }}$ for individual galaxies provided by redMaPPer. The spectroscopic membership fraction is larger than $P_{\text {mem }}$ for $0.05 \leqslant P_{\text {mem }} \leqslant 0.7$; conversely, the spectroscopic membership fraction is smaller than $P_{\text {mem }}$ at $P_{\text {mem }} \geqslant 0.8$. We compare spectroscopic richness estimates with redMaPPer richness estimates and find good agreement on average, but a range of a factor of two in spectroscopic richness at fixed redMaPPer richness. Overall, within the highrichness and low-redshift cut of our sample, spectroscopically estimated parameters such as velocity dispersion correlate well with photometric richness estimates, although the relations contain substantial scatter.
\end{abstract}

Key words: cosmology: observations - galaxies: clusters: general - galaxies: distances and redshifts - galaxies: kinematics and dynamics

Supporting material: machine-readable tables

\section{Introduction}

Cosmological models make different predictions for the comoving number density of clusters of fixed mass. The evolution of cluster abundances depends strongly on the amount of dark matter and dark energy in the universe. Thus, many groups have used different cluster mass proxies to determine the mass function and constrain cosmological parameters (e.g., Rines et al. 2007, 2008; Henry et al. 2009; Vikhlinin et al. 2009; Mantz et al. 2010; Rozo et al. 2010; Benson et al. 2013; Hasselfield et al. 2013; Planck Collaboration et al. 2014a, 2014b, 2016; Mantz et al. 2015; de Haan et al. 2016, and references therein).

The red sequence of cluster galaxies has been used by many investigators as a technique to discover new clusters (Gladders \& Yee 2000, 2005; Koester et al. 2007). One of the most sophisticated algorithms to detect clusters with the red sequence is the redMaPPer algorithm (Rykoff et al. 2014). Rykoff et al. (2014) apply the redMaPPer algorithm to imaging data from the Eighth Data Release (DR8) of the Sloan Digital Sky Survey (SDSS; Aihara et al. 2011). The redMaPPer cluster catalog includes an estimated richness of red-sequence galaxies that serves as a mass proxy. The redMaPPer richness parameter, $\lambda$, is an estimate of the number of red-sequence galaxies in a cluster above a luminosity limit $\left(0.2 L^{*}\right)$. The estimated value of $\lambda$ can include red-sequence galaxies from nearby line-of-sight structures (see an example in Sohn et al. 2018), and it does not include blue galaxies. Despite these limitations, comparisons with mock simulations indicate that $\lambda$ is a good proxy for mass, and one that requires only photometric data (Rykoff et al. 2014). Several tests show that redMaPPer richnesses correlate with other mass proxies, including the Sunyev-Zeldovich (SZ) signal (Rozo \& Rykoff 2014), X-ray luminosity (Rozo \& Rykoff 2014; Sadibekova et al. 2014), and a weak lensing signal (Simet et al. 2017). However, these comparisons usually are made either with samples of individual clusters selected by their properties at other wavelengths (e.g., clusters with SZ detections in the Planck catalog) or by stacking of clusters with the same richness parameter (e.g., Rykoff et al. 2008; Andreon 2014; Simet et al. 2017). If the parameter (e.g., richness) used to define cluster stacks has large intrinsic scatter (e.g., in true cluster mass), then the ensemble cluster samples only provide signals averaged over a broad range of intrinsic cluster properties.

Here, we compare optical richness estimates with dynamical mass estimates based on dense redshift surveys of individual clusters. Dynamical mass estimates have a long history, beginning with Zwicky $(1933,1937)$. In numerical simulations, either the virial theorem or the caustic technique can provide cluster mass estimates with little bias, but with some intrinsic scatter due to projection effects (Diaferio 1999; Evrard et al. 2008; Serra et al. 2011; Gifford \& Miller 2013; Mamon et al. 2013; Old et al. 2014). Hydrodynamical simulations show that the velocity distribution of galaxies is very similar to that of dark-matter particles (Faltenbacher \& Diemand 2006; Lau et al. 2010), with the possible exception of the brightest few galaxies (Lau et al. 2010; Wu et al. 2013). Thus, virial masses, caustic masses, or dynamical mass proxies such as velocity dispersion are a powerful test of richness-based mass estimates. 
We describe MMT/Hectospec spectroscopic observations of a sample of 27 clusters (six observed previously) selected with redMaPPer richness parameter $\lambda>64$ and redshift $z=0.08-0.25$. The redshift surveys of these clusters test the impact of projection effects on cluster identification and provide robust dynamical masses for comparison with the richness estimate $\lambda$. We extend our analysis to two additional redMaPPer clusters with Hectospec observations published here, and to an additional 94 clusters with velocity dispersions from previously published wide-field optical spectroscopy.

We discuss the cluster samples and spectroscopic data in Section 2. We measure the scaling relations in Section 3. We discuss the implications of our results in the context of other cosmological observations in Section 4. We assume a $\Lambda \mathrm{CDM}$ cosmology of $\Omega_{m}=0.3, \Omega_{\Lambda}=0.7$, and $H_{0}=$ $100 \mathrm{hm} \mathrm{s}^{-1} \mathrm{Mpc}^{-1}$ for all calculations.

\section{Observations}

The new observations presented here consist of optical spectroscopy acquired with the Hectospec instrument (Fabricant et al. 2005) on the MMT $6.5 \mathrm{~m}$ telescope. Hectospec provides simultaneous spectroscopy of up to 300 objects across a diameter of $1^{\circ}$. This telescope and instrument combination is ideal for studying the virial regions and outskirts of clusters at $z \lesssim 0.3$. Because cluster properties such as projected velocity dispersion depend on radius, wide-field spectroscopic coverage is important for measuring accurate global velocity dispersions and virial masses (Biviano et al. 2006).

In addition to the new spectra, we use extensive data from previous Hectospec cluster surveys (Rines et al. 2013, 2016), as well as from SDSS and NED. We divide our analysis into two samples (described in more detail below): the HeCS-red sample consists of redMaPPer-selected clusters exclusively, while an extended sample (HeCS-red-ext) adds clusters selected based on their X-ray or SZ properties.

We first describe the photometric and spectroscopic data and then provide details on the selection of the cluster samples.

\subsection{Optical Photometry and Spectroscopy}

All photometric data are taken from SDSS. We use composite model magnitudes, which are linear combinations of the fluxes of the best-fit exponential and deVaucouleurs radial profiles. We correct these magnitudes for Galactic extinction.

\subsubsection{Target Selection for New Spectroscopy: HeCS-red}

We use photometry primarily from the Tenth Data Release (DR10) of SDSS (Ahn et al. 2014) to identify targets for the new spectroscopic observations presented here. For two clusters (A0657 and RMJ072705+384613), inspection of DR10 photometry revealed that the catalogs contained "holes" around bright stars where the background subtraction may be problematic. We used DR7 photometry (which was less aggressive about removing regions around bright stars) to select targets for spectroscopy for objects in these holes. We acquired spectra with the Hectospec instrument (Fabricant et al. 2005) on the MMT $6.5 \mathrm{~m}$ telescope.

Our observing strategy closely matches the strategies used in the Hectospec Cluster Survey (HeCS; Rines et al. 2013) and the Hectospec Cluster Survey of SZ-selected clusters (HeCS-SZ; Rines et al. 2016). We used SDSS photometry within $3^{\prime}$ of the
Table 1

HeCS-red Redshifts from MMT/Hectospec

\begin{tabular}{|c|c|c|c|c|c|c|}
\hline \multicolumn{2}{|c|}{ Coordinates (J2000) } & \multirow{2}{*}{$z_{\odot}$} & \multirow{2}{*}{$\sigma_{z}$} & \multirow{2}{*}{$R_{\mathrm{XC}}$} & \multirow{2}{*}{ Flag } & \multirow{2}{*}{ Member } \\
\hline R.A. & Decl. & & & & & \\
\hline 0.01297 & 12.08851 & 0.201675 & 0.000088 & 16.75 & Q & 1 \\
\hline 0.01321 & 11.95804 & 0.267290 & 0.000227 & 6.07 & $\mathrm{Q}$ & 0 \\
\hline 0.02692 & 11.97140 & 0.142670 & 0.000165 & 6.38 & Q & 0 \\
\hline 0.03125 & 11.98484 & 0.203407 & 0.000126 & 10.55 & Q & 0 \\
\hline 0.03939 & 11.90404 & 0.129912 & 0.000044 & 9.07 & Q & 0 \\
\hline
\end{tabular}

Note. Only a portion of this table is shown here to demonstrate its form and content. A machine-readable version of the full table is available.

(This table is available in its entirety in machine-readable form.)

redMaPPer position to identify a red sequence in $g-r$ color and $r$-band apparent magnitude in each cluster field. We use composite model magnitudes corrected for Galactic extinction. For each cluster, we then identify a cutoff in apparent magnitude depending on the redshift and richness of the target cluster. Specifically, for each cluster, we choose a limiting magnitude that offers a good compromise of high completeness (sparser targets produce fewer fiber conflicts) and dense sampling. The limiting apparent magnitude ranges from $r=20.0$ (low-redshift, rich cluster) to $r=21.2$ (higherredshift, less rich cluster). Targets are primarily drawn from galaxies with $g-r$ colors within 0.2 mag of the red sequence (observed-frame colors), and we assign higher priorities to brighter galaxies and galaxies closer to the cluster center. This approach provides reasonably high sampling in the cluster cores, but can lead to relatively sparse sampling of dense regions outside the core. Experience from HeCS and HeCS-SZ indicates that two Hectospec configurations yield fairly high completeness for bright galaxies in cluster virial regions for samples of 700-800 primary target galaxies. We included galaxies with slightly bluer colors (up to 0.4 mag bluer than the red sequence) as targets to fill any unused fibers. We matched all targets to redshifts from the literature as compiled by NED ${ }^{6}$ as of 2015 September as well as to SDSS DR10 spectra. Most of the targets with existing redshifts are from SDSS, but several are from targeted studies of individual clusters (e.g., Abraham et al. 1996; Barrena et al. 2007b, for A2390 and A0115 respectively). We removed targets with existing redshifts from the targeting catalogs prior to fiber assignment. After observations were complete, we supplemented the redshift catalogs with additional redshifts from SDSS DR13 (Albareti et al. 2017) that were unavailable at the time of target selection.

Table 1 lists 10,589 new redshifts measured with Hectospec in the fields of 23 clusters. We visually inspected all spectra to confirm the reliability of the redshifts. Column 5 of Table 1 lists the cross-correlation score $R_{\mathrm{XC}}$ from the IRAF package rvsao (Kurtz \& Mink 1998). A score of $R_{\mathrm{XC}}>3$ indicates a reliable redshift; some galaxies with smaller values of $R_{\mathrm{XC}}$ are included when visual inspection shows multiple obvious absorption and/or emission lines and the spectrum suffers from contamination (e.g., light bleeding into the spectrum from a nearby fiber containing a bright star). The results of visual inspection are listed as a Flag with possible values Q (unambiguous redshift), ? (medium-confidence redshift), and

\footnotetext{
6 http://ned.ipac.caltech.edu
} 
Table 2

HeCS-red Members from the Literature Redshifts

\begin{tabular}{lcccc}
\hline \hline \multicolumn{2}{c}{ Coordinates (J2000) } & & $\sigma_{\odot}$ & References \\
\cline { 1 - 2 } R.A. & Decl. & & & \\
\hline 0.41523 & 12.05312 & 0.203698 & 0.000043 & 1 \\
0.41633 & 12.06724 & 0.204635 & 0.000060 & 1 \\
0.41782 & 11.92581 & 0.199108 & 0.000020 & 1 \\
0.43310 & 12.06504 & 0.201360 & 0.000067 & 1 \\
0.43693 & 12.06558 & 0.201616 & 0.000030 & 1 \\
\hline
\end{tabular}

Note. References: (1) SDSS, (2) NED Only a portion of this table is shown here to demonstrate its form and content. A machine-readable version of the full table is available.

(This table is available in its entirety in machine-readable form.)

X (low-confidence redshift). Table 2 lists redshifts from SDSS and other literature (as compiled by NED) for galaxies classified as cluster members by the caustic technique (see below). Prior to the Hectospec observations, we measured three redshifts of bright galaxies with the FAST instrument (Fabricant et al. 1998) on the $1.5 \mathrm{~m}$ Tillinghast telescope at the Fred Lawrence Whipple Observatory. Two of these galaxies were observed with Hectospec with concordant redshifts, the third galaxy is at coordinates $(\alpha, \delta)=$ $(23: 26: 26.15,+29: 21: 52.67)$ and has heliocentric redshift $c z_{\odot}=(67891.9 \pm 75) \mathrm{km} \mathrm{s}^{-1}$. This galaxy is a member of RMJ2326.

Figures 1 and 2 show redshift histograms of the HeCS-red clusters. Each of the clusters in the sample shows a prominent peak in the redshift histogram close to the location of the redMaPPer photometric redshift. Several clusters (A0586, A0098S, RMJ0751, RMJ2201, RMJ2326, RMJ0830, and RMJ0826) show additional peaks that could contaminate the richness estimates.

\subsubsection{Previous Spectroscopy: CIRS, HeCS, and HeCS-SZ}

HeCS-red-ext is an extended sample that adds clusters selected based on their X-ray or SZ properties to the HeCS-red sample.

The Cluster Infall Regions in SDSS (CIRS) program (Rines \& Diaferio 2006) studied the spectroscopic properties of 72 X-ray selected clusters using data from the Fourth Data Release of SDSS (Adelman-McCarthy et al. 2006). These galaxies are primarily in the Main Galaxy Sample and thus the cluster redshift surveys are reasonably complete to $r=17.77$.

$\mathrm{HeCS}$ is a spectroscopic survey of 58 galaxy clusters at moderate redshift $(z=0.1-0.3)$ with $\mathrm{MMT} /$ Hectospec. HeCS clusters were selected based on X-ray flux.

For HeCS spectroscopy, we used the red sequence to preselect likely cluster members as primary targets (targets within \pm 0.3 mag of the red sequence). We then filled otherwise unassigned fibers with bluer targets (Rines et al. 2013 describes the details of target selection).

HeCS-SZ includes clusters with $z<0.2$ selected based on integrated Compton parameter measurements from the Planck satellite (Planck Collaboration et al. 2014b) and photometry from SDSS DR10. The spectroscopic observing strategy for HeCS-SZ closely matches HeCS. Figure 14 of Rines et al. (2013) shows that the vast majority of cluster members are within $0.1 \mathrm{mag}$ of the red sequence. Based on this result, we narrowed the color selection for primary targets in HeCS-SZ to \pm 0.2 mag to improve the yield of cluster members. We used this narrowed color selection for selecting targets for HeCS-red observations as well.

\subsection{Cluster Sample Selection}

Previous surveys (Rines \& Diaferio 2006; Rines et al. 2013, 2016) contain spectroscopy for several clusters in the redMaPPer catalog. However, these clusters were selected based on their X-ray or SZ properties. As a result, scaling relations based on these samples could be biased relative to the scaling relations for a purely redMaPPer-selected sample.

We define a redMaPPer-selected sample of 30 clusters covering redshift $0.10 \leqslant z \leqslant 0.25$, redMaPPer richness parameter $\lambda \geqslant 64$, coordinates $\delta>10^{\circ}$, and either $\alpha<9 h$ or $\alpha>20 h$ (Figure 3). The redMaPPer catalog extends to $\lambda=20$ and $z=0.55$ (Rykoff et al. 2014); the sample we select is limited to the richest clusters at low redshift.

Figure 3 shows the selection of the HeCS-red sample. Although these clusters were selected based on their redMaPPer richness, two of the newly observed clusters (A115, A2390) lie above the X-ray limit of HeCS and the SZ limit of HeCS-SZ but were not included in those surveys.

Among the 30 clusters meeting the criteria for HeCS-red, 1 cluster (A655) was observed with Hectospec for the Hectospec Cluster Survey (HeCS; Rines et al. 2013) and 5 clusters (A586, A98S, A2409, A7, and A2443) were observed with Hectospec for the Hectospec Cluster Survey of SZ-Selected clusters (HeCS-SZ; Rines et al. 2016). We observed 21 additional clusters for HeCS-red, yielding a redMaPPer-selected cluster sample of 27 clusters that is $90 \%$ complete. Hereafter we call this sample of 27 redMaPPer-selected clusters HeCS-red.

We also observed one cluster, RMJ023054.9+024719.6 (with $\lambda=90.7 \pm 4.1$, from the declination range $0^{\circ}<\delta<10^{\circ}$. We also include Hectospec data for A2355 $(\lambda=109.4 \pm 4.8)$ in the same declination range, originally selected based on its SZ signal from the Planck satellite (Planck Collaboration et al. 2014b). Because even the central redshift of A2355 was poorly known at the time of observation (e.g., Böhringer et al. 2004, lists a redshift of $z=0.1244$ ), spectroscopic targets for A2355 were selected solely by apparent magnitude with no color cuts. RMJ023054.9 +024719.6 and A2355 are included in the HeCS-red-ext sample and excluded from the HeCS-red sample. Figure 4 shows redshift histograms and phase space diagrams of these two clusters. In total, HeCS-red-ext includes 121 clusters in a heterogeneously selected sample.

\subsection{Membership, Velocity Dispersions, and Masses}

The caustic technique (Diaferio \& Geller 1997; Diaferio 1999; Serra et al. 2011) isolates cluster members from foreground and background galaxies in phase space. The infall regions of clusters produce well-defined envelopes in the redshift-projected radius diagram that contain the vast majority of cluster members. The edges of these envelopes are determined by first adaptively smoothing the galaxy distribution in phase space and then determining the rest-frame velocity where the density of galaxies increases rapidly. The edges of the envelopes are called caustics and they are related to the escape velocity profile of the cluster (see Diaferio 2009; Serra et al. 2011, for reviews). In numerical simulations, $96 \%$ of cluster members within $r_{200}$ lie inside the caustic envelope, and only $2 \%$ of galaxies inside the caustic envelope are actually interlopers. Within the larger radius $3 r_{200}$, 


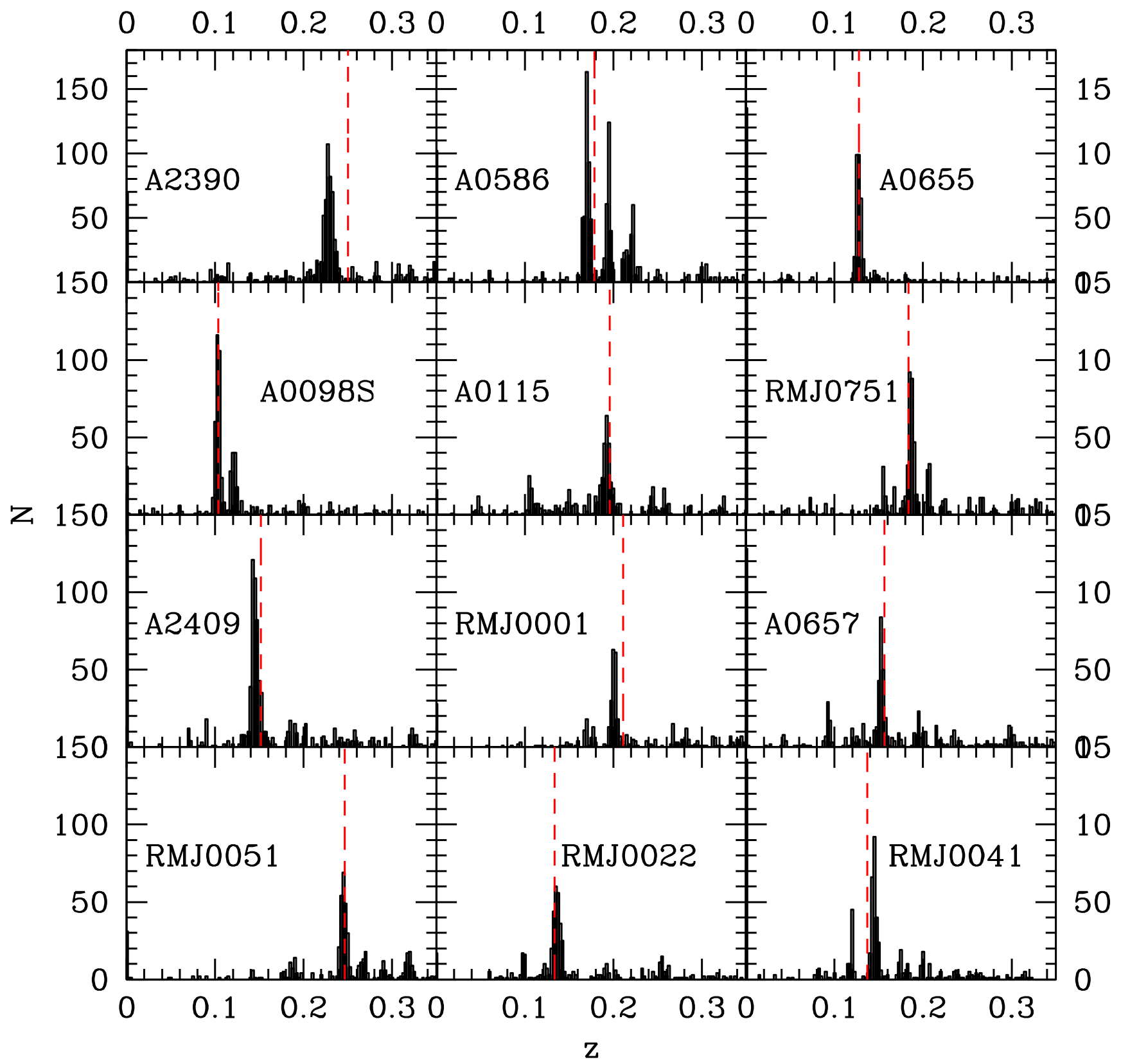

Figure 1. Redshift histograms for galaxies within $0.5^{\circ}$ of the cluster centers for the redMaPPer sample. Bins have width $\Delta z=0.0025$. Red dashed lines indicate the photometric redshifts from redMaPPer.

where the caustic technique is the only usable method, the completeness is $95 \%$ and the interloper fraction is $8 \%$ (Serra \& Diaferio 2013). The escape velocity profile is the basis for a mass profile that can extend into the infall region where the galaxies are gravitationally bound but not virialized. Caustic mass estimates generally agree with estimates from X-ray observations and gravitational lensing (e.g., Biviano \& Girardi 2003; Rines et al. 2003, 2007; Diaferio et al. 2005; Rines \& Diaferio 2006; Geller et al. 2013; Maughan et al. 2016, and references therein).

Figures 5 and 6 show the phase space diagrams of the HeCSred clusters not already published in HeCS or HeCS-SZ. All clusters display infall patterns; the caustics are shown on the figures. Clusters are ordered by decreasing richness parameter $\lambda$. There is a general trend of decreasing central velocity dispersion with decreasing richness. Figure 4 shows the phase space diagrams for the HeCS-red-ext clusters included in Table 1.

We apply the prescription of Danese et al. (1980) to determine the mean redshift $c z_{\odot}$ and projected velocity dispersion $\sigma_{p}$ of each cluster from all galaxies within the caustics. We calculate $\sigma_{p}$ using only the cluster members projected within $r_{200}$ estimated from the caustic mass profile. Note that our measured velocity dispersions use the caustic technique only to define membership and the limiting radius $r_{200}$. Independent of its performance as a mass estimator, the caustic technique is a highly efficient membership selection algorithm, especially at the relatively small radii we focus on here (Serra \& Diaferio 2013). Table 3 lists the central cluster redshifts, velocity dispersions inside $r_{200}$, and $M_{200}$ from the caustic mass profile. The eighth column of Table 3 indicates 


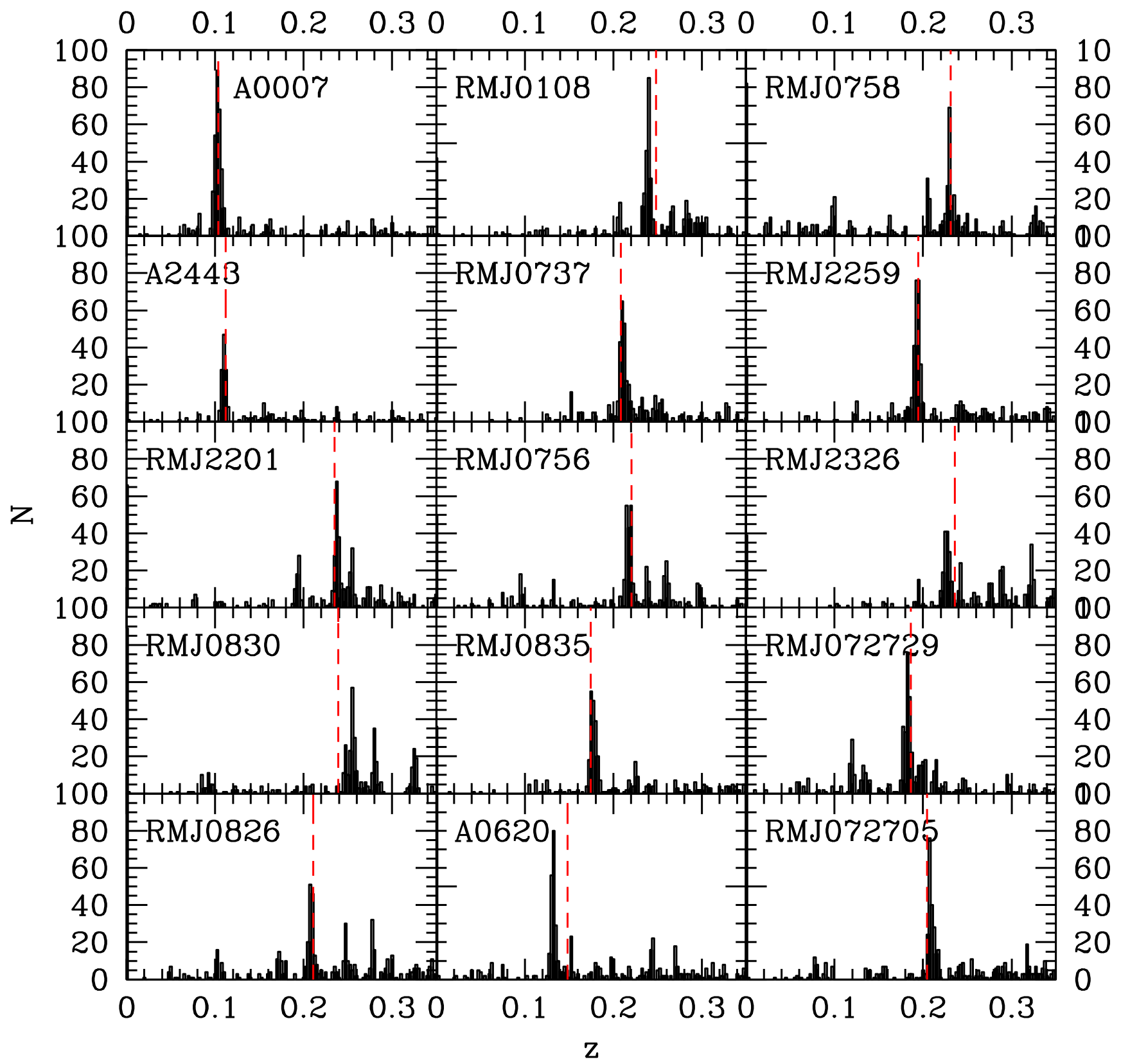

Figure 2. Redshift histograms for galaxies within $0.5^{\circ}$ of the cluster centers for the redMaPPer sample. Bins have width $\Delta z=0.0025$. Red dashed lines indicate the photometric redshifts from redMaPPer.

whether the cluster is part of the CIRS, HeCS, HeCS-SZ, or HeCS-red sample.

The uncertainties in caustic masses mostly originate from projection effects, because the caustic method assumes spherical symmetry, whereas clusters generally are triaxial. Numerical simulations show that the algorithm to estimate the uncertainties in the mass, suggested by Diaferio (1999) and Serra et al. (2011), that we also adopt here, returns, on average, the $50 \%$ confidence range of the deviation, due to projection effects, of the measured mass profile from the true profile (see Figure 16 in Serra et al. 2011). The algorithm is based on the ratio between the number of galaxies outside and within the caustics: the small uncertainties in some caustic mass estimates come from clusters where the redshift diagram is particularly clean and the number of galaxies outside the caustics is low; consequently, both the caustic location and the derived caustic mass appear to be solid. Clearly, the estimated uncertainty is reliable only in a statistical sense; in some individual cases, when an unfortunate projection produces, at the same time, a clean redshift diagram and an incorrect caustic amplitude, it can happen that the discrepancy between the true and the estimated masses is larger than the estimated uncertainty: unfortunately, the frequency of the occurrence of these cases currently is difficult to estimate, because the redshift diagrams of real clusters tend to be substantially cleaner than the diagrams of mock clusters extracted from $N$-body simulations (Diaferio 1999), probably due to the uncertainties in the galaxy formation modeling (e.g., Knebe et al. 2018).

\subsection{Comparison of Spectroscopic Redshifts with redMaPPer Photometric Redshifts}

The photometric redshifts in the redMaPPer catalog are usually close to the central redshifts we obtain in our 
Table 3

Dynamical Mass and Richness Estimates

\begin{tabular}{|c|c|c|c|c|c|c|c|c|}
\hline Cluster & $\begin{array}{c}\alpha \\
\operatorname{deg}\end{array}$ & $\begin{array}{c}\delta \\
\operatorname{deg}\end{array}$ & $z_{\odot}$ & $\begin{array}{c}\sigma_{p} \\
\mathrm{~km} \mathrm{~s}^{-1}\end{array}$ & $\begin{array}{c}M_{200, c} \\
10^{14} M_{\odot}\end{array}$ & $\lambda$ & Spectra & redMaPPer ID \\
\hline A2390 & 328.39839 & 17.69735 & 0.2283 & $1278_{-55}^{+64}$ & $12.10 \pm 0.62$ & $149.3 \pm 5.5$ & HeCS-red & RMJ215336.8+174143.7 \\
\hline A0586 & 113.09431 & 31.62882 & 0.1702 & $797_{-45}^{+55}$ & $4.34 \pm 0.18$ & $145.1 \pm 5.5$ & HeCS-SZ & RMJ073220.3+313800.7 \\
\hline A0655 & 126.34018 & 47.15855 & 0.1271 & $777_{-47}^{+58}$ & $3.33 \pm 0.16$ & $130.7 \pm 5.4$ & $\mathrm{HeCS}$ & RMJ082529.1+470800.9 \\
\hline A0098S & 11.63442 & 20.47665 & 0.1038 & $624_{-38}^{+47}$ & $2.42 \pm 0.11$ & $127.1 \pm 3.3$ & HeCS-SZ & RMJ004629.3+202804.8 \\
\hline A0115 & 14.00240 & 26.33962 & 0.1916 & $1176_{-59}^{+70}$ & $10.60 \pm 0.16$ & $111.5 \pm 3.7$ & HeCS-red & RMJ005600.3+262032.3 \\
\hline RMJ075100.8+173753.8 & 117.81519 & 17.65724 & 0.1863 & $637_{-51}^{+68}$ & $1.72 \pm 0.07$ & $110.5 \pm 6.1$ & HeCS-red & RMJ075100.8+173753.8 \\
\hline A2409 & 330.22102 & 20.96189 & 0.1450 & $1038_{-64}^{+79}$ & $5.30 \pm 0.38$ & $92.8 \pm 4.6$ & HeCS-SZ & RMJ220052.6+205809.3 \\
\hline RMJ000158.5+120358.0 & 00.50730 & 12.07572 & 0.2010 & $647_{-51}^{+67}$ & $2.38 \pm 0.22$ & $84.9 \pm 3.5$ & HeCS-red & RMJ000158.5+120358.0 \\
\hline A0657 & 125.83242 & 15.95854 & 0.1524 & $777_{-50}^{+62}$ & $3.01 \pm 0.39$ & $81.6 \pm 3.6$ & HeCS-red & RMJ082319.3+155745.8 \\
\hline RMJ005105.2+261716.7 & 12.76264 & 26.30037 & 0.2454 & $661_{-51}^{+66}$ & $2.97 \pm 0.05$ & $81.6 \pm 4.0$ & HeCS-red & RMJ005105.2+261716.7 \\
\hline RMJ002224.7+231733.0 & 05.59923 & 23.29194 & 0.1365 & $710_{-41}^{+49}$ & $3.51 \pm 0.21$ & $80.8 \pm 3.3$ & HeCS-red & RMJ002224.7+231733.0 \\
\hline RMJ004118.5+252609.1 & 10.31908 & 25.43062 & 0.1447 & $652_{-49}^{+64}$ & $2.24 \pm 0.06$ & $80.0 \pm 3.5$ & HeCS-red & RMJ004118.5+252609.1 \\
\hline A0007 & 02.94185 & 32.42523 & 0.1030 & $816_{-46}^{+55}$ & $3.03 \pm 1.28$ & $79.3 \pm 4.1$ & HeCS-SZ & RMJ001145.3+322456.4 \\
\hline RMJ010819.0+275802.1 & 17.07981 & 27.96756 & 0.2393 & $609_{-58}^{+81}$ & $1.55 \pm 0.02$ & $78.1 \pm 3.7$ & HeCS-red & RMJ010819.0+275802.1 \\
\hline RMJ075822.7+264120.6 & 119.62574 & 26.68098 & 0.2298 & $986_{-64}^{+80}$ & $7.50 \pm 0.21$ & $77.9 \pm 4.0$ & HeCS-red & RMJ075822.7+264120.6 \\
\hline A2443 & 336.50488 & 17.37134 & 0.1102 & $652_{-46}^{+58}$ & $1.93 \pm 0.64$ & $77.6 \pm 2.8$ & HeCS-SZ & RMJ222607.9+172123.4 \\
\hline RMJ073720.9+351741.7 & 114.33470 & 35.28469 & 0.2109 & $602_{-48}^{+63}$ & $2.80 \pm 0.25$ & $76.6 \pm 4.3$ & HeCS-red & RMJ073720.9+351741.7 \\
\hline RMJ225946.5+310223.9 & 344.95589 & 31.03735 & 0.1937 & $661_{-48}^{+61}$ & $2.60 \pm 0.03$ & $75.2 \pm 3.5$ & HeCS-red & RMJ225946.5+310223.9 \\
\hline RMJ220107.7+111805.2 & 330.28072 & 11.29804 & 0.2379 & $560_{-54}^{+77}$ & $1.41 \pm 0.15$ & $74.1 \pm 4.4$ & HeCS-red & RMJ220107.7+111805.2 \\
\hline RMJ075655.8+383933.2 & 119.26340 & 38.68267 & 0.2172 & $683_{-63}^{+87}$ & $1.99 \pm 0.02$ & $72.7 \pm 3.7$ & HeCS-red & RMJ075655.8+383933.2 \\
\hline RMJ083056.4+322412.2 & 127.69104 & 32.45600 & 0.2551 & $482_{-51}^{+75}$ & $0.88 \pm 0.12$ & $67.6 \pm 4.1$ & HeCS-red & RMJ083056.4+322412.2 \\
\hline RMJ083513.0+204654.9 & 128.76012 & 20.78112 & 0.1770 & $611_{-49}^{+64}$ & $2.22 \pm 0.02$ & $67.5 \pm 4.2$ & HeCS-red & RMJ083513.0+204654.9 \\
\hline RMJ232626.2+292152.7 & 351.60948 & 29.35085 & 0.2273 & $938_{-73}^{+95}$ & $3.63 \pm 0.14$ & $68.9 \pm 5.6$ & HeCS-red & RMJ232626.2+292152.7 \\
\hline RMJ072729.3+422756.1 & 111.88434 & 42.51032 & 0.1828 & $702_{-48}^{+60}$ & $3.04 \pm 0.14$ & $66.9 \pm 3.8$ & HeCS-red & RMJ072729.3+422756.1 \\
\hline RMJ082657.6+310804.9 & 126.72990 & 31.14427 & 0.2088 & $890_{-67}^{+86}$ & $6.08 \pm 0.32$ & $65.7 \pm 3.2$ & HeCS-red & RMJ082657.6+310804.9 \\
\hline A0620 & 121.43548 & 45.67952 & 0.1316 & $702_{-47}^{+59}$ & $2.57 \pm 0.07$ & $64.3 \pm 2.5$ & HeCS-red & RMJ080543.3+454058.9 \\
\hline RMJ072705.2+384613.4 & 111.77754 & 38.80076 & 0.2084 & $614_{-46}^{+59}$ & $2.35 \pm 0.04$ & $64.0 \pm 3.8$ & HeCS-red & RMJ072705.2+384613.4 \\
\hline RMJ023054.9+024719.6 & 37.72578 & 02.78778 & 0.2425 & $878_{-61}^{+77}$ & $4.34 \pm 0.92$ & $90.7 \pm 4.1$ & HeCS-red-ext & RMJ023054.9+024719.6 \\
\hline A2355 & 323.81759 & 01.39962 & 0.2306 & $911_{-88}^{+125}$ & $5.12 \pm 0.73$ & $109.4 \pm 4.8$ & HeCS-red-ext & RMJ213518.8+012527.0 \\
\hline
\end{tabular}

Note. Redshift $z$ and velocity dispersion $\sigma_{p}$ are computed for galaxies defined as members using the caustics.

(This table is available in machine-readable form.)

hierarchical clustering analysis of the cluster redshifts (see D99 for details). However, for about half of the clusters, our central redshifts differ by more than a percent from the redMaPPer photometric redshifts.

Figure 7 compares photometric redshift estimates from the redMaPPer catalog with the spectroscopic redshifts. For the HeCS-red sample, the mean offset is $z_{\text {spec }}-z_{\mathrm{RM}}=-0.0019 \pm$ 0.0014 . For HeCS-red-ext, the mean offset is $z_{\text {spec }}-z_{\mathrm{RM}}=$ $-0.0028 \pm 0.0005$ (green line in Figure 7), suggesting a bias in the photometric redshifts at $5.5 \sigma$ confidence level. Comparisons of redMaPPer redshifts to BCG redshifts in DR8 showed no such offset (Rykoff et al. 2014). Studies of BCG redshifts relative to their clusters shows that, while most BCGs are located close to the mean redshift of their clusters, some BCGs have significant velocity offsets (e.g., Lauer et al. 2014).

The redshift bias observed for the HeCS-red-ext sample could be produced by an excess of background contamination over foreground contamination. The photometric redshift window surrounding the cluster contains a larger volume at redshifts above than the cluster redshift than the volume below than the cluster redshift.

Spectroscopic targets for A2355 were selected by apparent magnitude only (no color cut). Figure 4 shows that this cluster is not as well sampled as clusters of similar richness where target selection prioritized red-sequence galaxies. This difference highlights the greater efficiency of red-sequence target selection for identifying cluster members.

\section{Cluster Scaling Relations}

\subsection{Bayesian Parameter Estimation}

We determine the scaling relations between redMaPPer cluster richness $\lambda$ and spectroscopic properties for the HeCS-red sample using a Bayesian approach similar to HeCS-SZ. A number of unknown hidden variables produces a scatter in the linear correlation $Y=a+b X$. We model this scatter with a single parameter, the intrinsic dispersion $\sigma_{\text {int }}$. Therefore, given a measure $X_{i}$ with uncertainty $\sigma_{X_{i}}$, the probability of measuring $Y_{i}$ with uncertainty $\sigma_{Y_{i}}$ is $p\left(Y_{i}, \sigma_{Y_{i}} \theta, X_{i}, \sigma_{X_{i}}\right)$, where $\theta=\left\{a, b, \sigma_{\text {int }}\right\}$. We assume the Gaussian likelihood

$$
p(D \mid \theta, M)=\prod_{i} \frac{1}{\left(2 \pi \sigma_{i}^{2}\right)^{1 / 2}} \exp \left[\frac{-\left(Y_{i}-a-b X_{i}\right)^{2}}{2 \sigma_{i}^{2}}\right],
$$




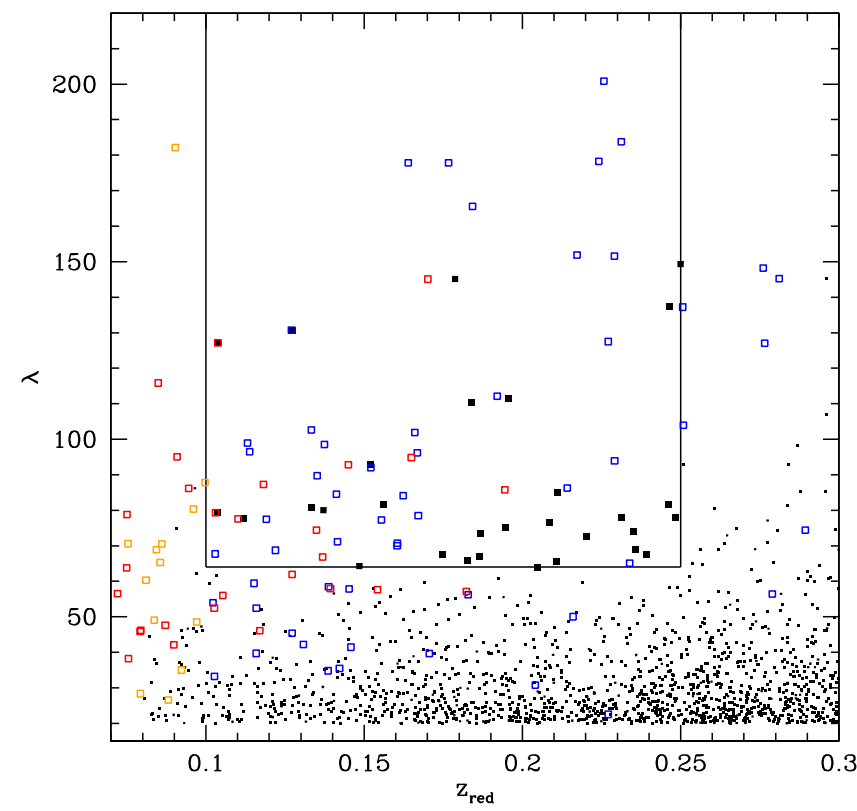

Figure 3. redMaPPer richness estimates $\lambda$ vs. redshift. Small black squares show clusters within the spatial footprint of our redMaPPer-selected target sample. Solid lines show the redshift and richness limits of the target sample. Red squares show clusters in the HeCS-red sample and triangles show clusters from the extended HeCS-red-ext sample (including clusters from X-ray and SZ-selected samples).

where $M$ is the model with parameter set $\theta, D$ is the data, and

$$
\sigma_{i}^{2}=\sigma_{\text {int }}^{2}+\sigma_{Y_{i}}^{2}+b^{2} \sigma_{X_{i}}^{2} .
$$

We assume independent flat priors for both $a$ and $b$. For the intrinsic dispersion $\sigma_{\mathrm{int}}$, which is positive defined, we assume

$$
p\left(\sigma_{\text {int }} \mid M\right)=\frac{\mu^{r}}{\Gamma(r)} x^{r-1} \exp (-\mu x),
$$

where $x=1 / \sigma_{\text {int }}^{2}$, and $\Gamma(r)$ is the usual gamma function. This PDF describes a variate with mean $r / \mu$, and variance $r / \mu^{2}$. We set $r=\mu=10^{-5}$, which guarantees an almost flat prior.

We estimate the parameter PDF $p(\theta \mid D, M)$ via Markov Chain Monte Carlo (MCMC) sampling with the code APEMoST (Buchner \& Gruberbauer 2011; Gruberbauer et al. 2009). We obtain a fairly complete sampling with $2 \times 10^{6} \mathrm{MCMC}$ iterations. The boundaries of the parameter space were set to $[-100,100]$ for $a$ and $b$, and $[0.01,100]$ for $\sigma_{\text {int }}$. As the three best-fit parameters, $a$, $b$, and $\sigma_{\text {int }}$ of the Bayesian analysis, we adopt the medians derived from the posterior PDF $p(\theta \mid D, M)$. Likewise, we adopt the boundaries of the $68 \%$ credible intervals around the medians as the uncertainties on these best-fit parameters. Table 4 lists the best-fit parameters.

\subsection{Scaling of Richness with Velocity Dispersion and Dynamical Mass}

Figure 8 shows the best-fit relation for $P\left(\sigma_{p} \mid \lambda\right)$, the expected velocity dispersion at fixed richness proxy $\lambda$. Substantial scatter is present at fixed $\lambda$; the range of measured velocity dispersion at fixed $\lambda$ is a factor of two. Figure 9 shows $\sigma_{p}$ versus $\lambda$ for the enlarged HeCS-red-ext sample. The best-fit relation for the HeCS-red-ext sample agrees with the best-fit relation for the HeCS-red sample, indicating that sample selection does not bias the inferred parameters of the scaling relation, at least to

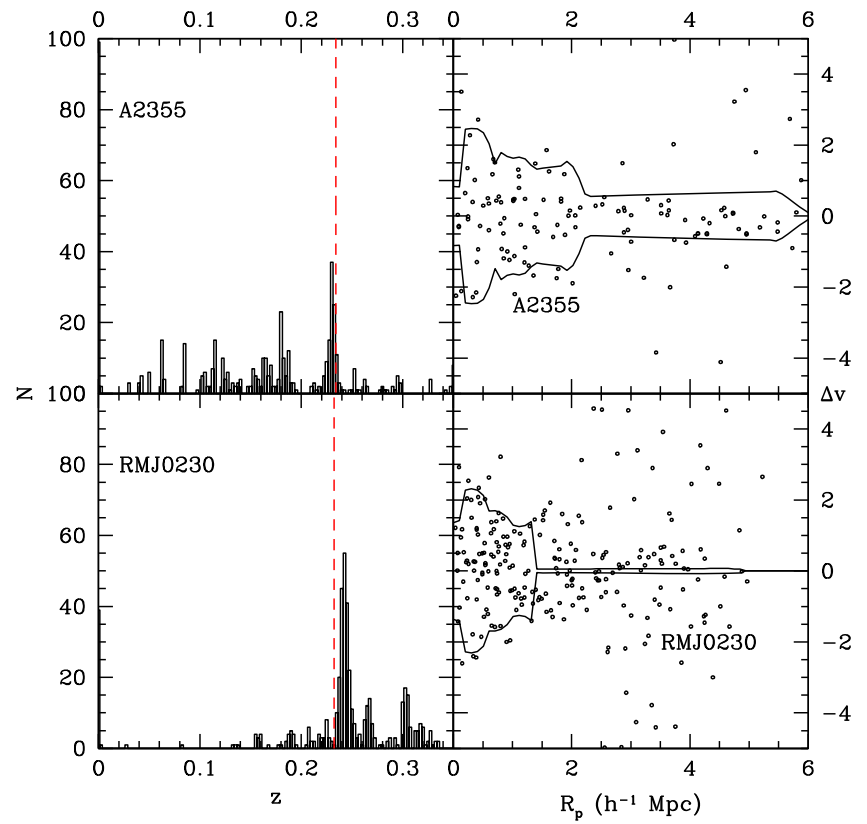

Figure 4. Similar to Figures 1 and 5, but for the HeCS-red-ext clusters A2355 and RMJ0230 with redshifts in Table 1. Note that the target catalog for A2355 did not use a color cut.

the accuracy of the samples presented here. However, the enlarged sample contains more outliers, and the range of measured velocity dispersion at fixed $\lambda$ increases to a factor of three.

Figure 10 shows the best-fit relation $P\left(M_{200} \mid \lambda\right)$, the caustic mass $M_{200}$ obtained at fixed $\lambda$, for the HeCS-red sample. Figure 11 shows the best-fit relation for the HeCS-red-ext sample. The intrinsic scatter in this relation is about $90 \%$ for both the HeCS-red and HeCS-red-ext samples (Table 4). Similar to the $\sigma_{p}-\lambda$ scaling relation, there is no evidence of bias in the inferred parameters for the HeCS-red-ext sample due to the heterogeneous sample selection. The range of $M_{200}$ at fixed $\lambda$ is about a factor of 10 (20) for the HeCS-red (HeCS-red-ext) sample, consistent with scaling the range of measured velocity dispersions at fixed $\lambda$ by a virial scaling relation $M_{200} \propto \sigma_{p}^{3}$. Thus, the estimated richness $\lambda$ is a low-precision predictor of the measured values $\sigma_{p}$ or $M_{200}$ for individual clusters.

\subsection{Outliers}

The scaling relations show that many clusters are outliers from the main relation. Outliers can be produced by large overestimates or underestimates of either richness or velocity dispersion or by the intrinsic scatter in the scaling relation. We use the scaling relation of $P\left(\sigma_{p} \mid \lambda\right)$ for the HeCS-red-ext sample to identify possible outliers. Specifically, we look at clusters that lie more than $\sigma_{\text {int }}$ away from this relation. Notably, all four outliers from HeCS-red with small $\sigma_{p}$ given their richness (A98S, RMJ0751, RMJ0830, and RMJ2201) show a secondary peak at slightly higher redshift $\left(z_{\text {secondary }}-z_{\text {red }}<0.04\right.$; see Figures 1 and 2). This result suggests that the richnesses of these clusters may be overestimated due to nearby background structure included within the photometric redshift window.

We provide additional information on some of the HeCS-red clusters below. 


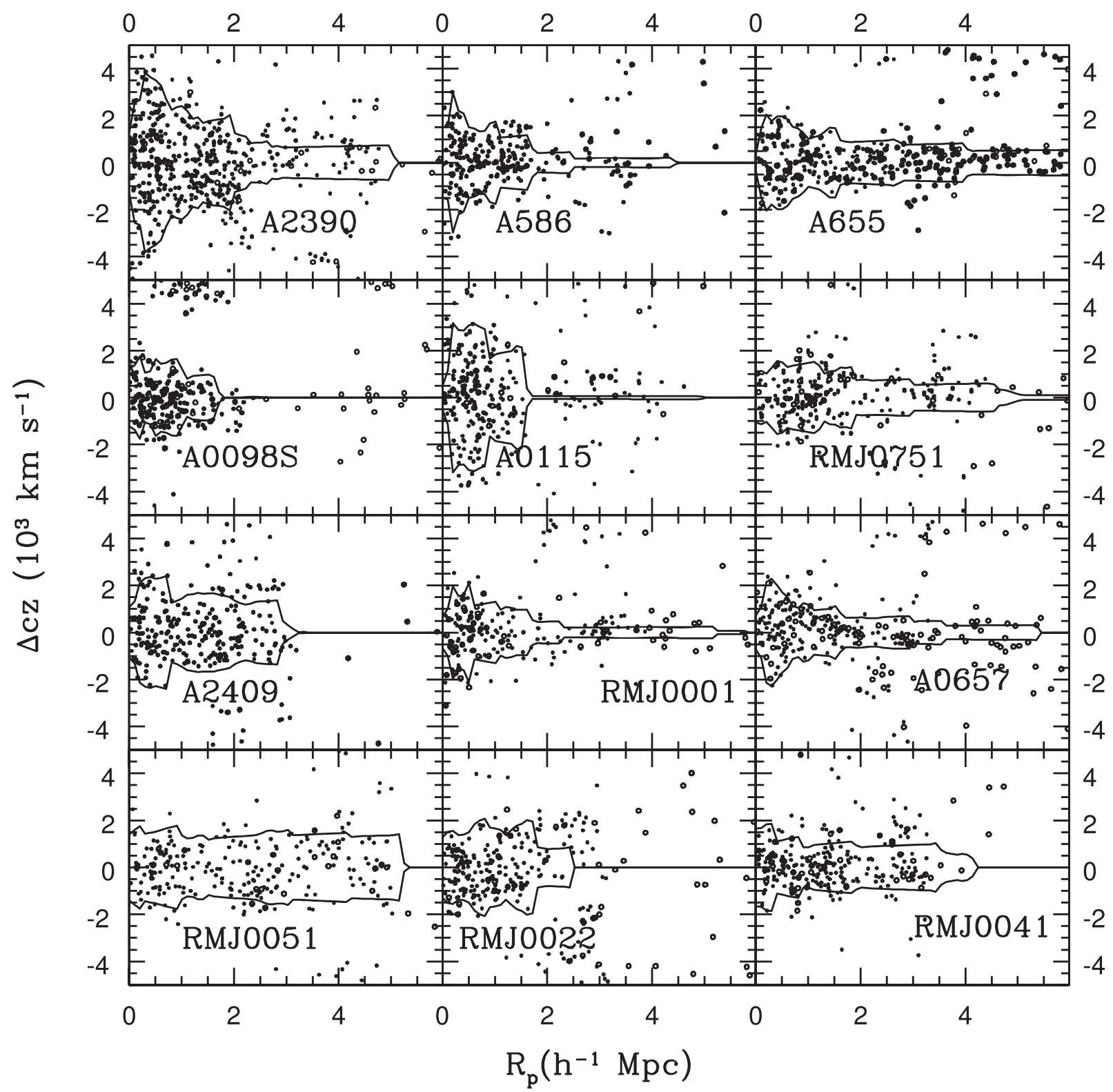

Figure 5. Redshift (rest-frame clustrocentric velocity) vs. projected radius for galaxies around HeCS-red clusters. The caustic pattern is evident as the trumpet-shaped regions with high density. The solid lines indicate our estimate of the location of the caustics in each cluster. Clusters are ordered left to right and top to bottom by decreasing richness parameter $\lambda$.

1. A98S. This system is the southern component of the double cluster A98N/A98S (Forman et al. 1981). The two X-ray peaks are separated by 8.9 or $0.8 h^{-1} \mathrm{Mpc}$ on the sky. Chandra observations show some evidence that A98N/A98S are in the early stages of merging (PaternoMahler et al. 2014), while a third cluster, termed A98SS, lies $1.0 \mathrm{~h}^{-1} \mathrm{Mpc}$ South of A98S in projection. Beers et al. (1982) used redshifts of 24 member galaxies in A98N/ A98S to study the system as a two-body merger and conclude that a bound-ingoing model is most probable. Using a larger redshift sample, Paterno-Mahler et al. (2014) confirm that A98N/A98S can be modeled as a two-body, bound-ingoing model, although an unboundoutgoing scenario is also consistent with the data. They further report that A98SS is not bound to A98S. Although A98N has a larger X-ray luminosity than A98S (Forman et al. 1981; Jones \& Forman 1999), the X-ray temperature of A98N is only marginally larger than A98S (PaternoMahler et al. 2014), and A98S contains more galaxies than A98N. There is a bright galaxy at the center of both A98N and A98S. There are 39 (22) spectroscopically confirmed members within $4 ! 4$ of the bright galaxy at the center of A98S $(\mathrm{A} 98 \mathrm{~N})$. The galaxies in $\mathrm{A} 98 \mathrm{~N}$ are separated by $-435 \pm 252 \mathrm{~km} \mathrm{~s}^{-1}$ from the galaxies in A98S. The velocity difference from our larger sample is somewhat smaller than found by Paterno-Mahler et al. (2014). This difference shifts the possible unboundoutgoing scenario for A98N/A98S to a bound-outgoing scenario. Because the redshifts of $\mathrm{A} 98 \mathrm{~N}$ and A98S overlap, we treat the merging clusters A98N/A98S as a single massive system.

With our larger redshift sample, we confirm that A98SS is separated from A98S by $\Delta v \sim 5000 \mathrm{~km} \mathrm{~s}^{-1}$ (visible near the top of the phase space diagram of 


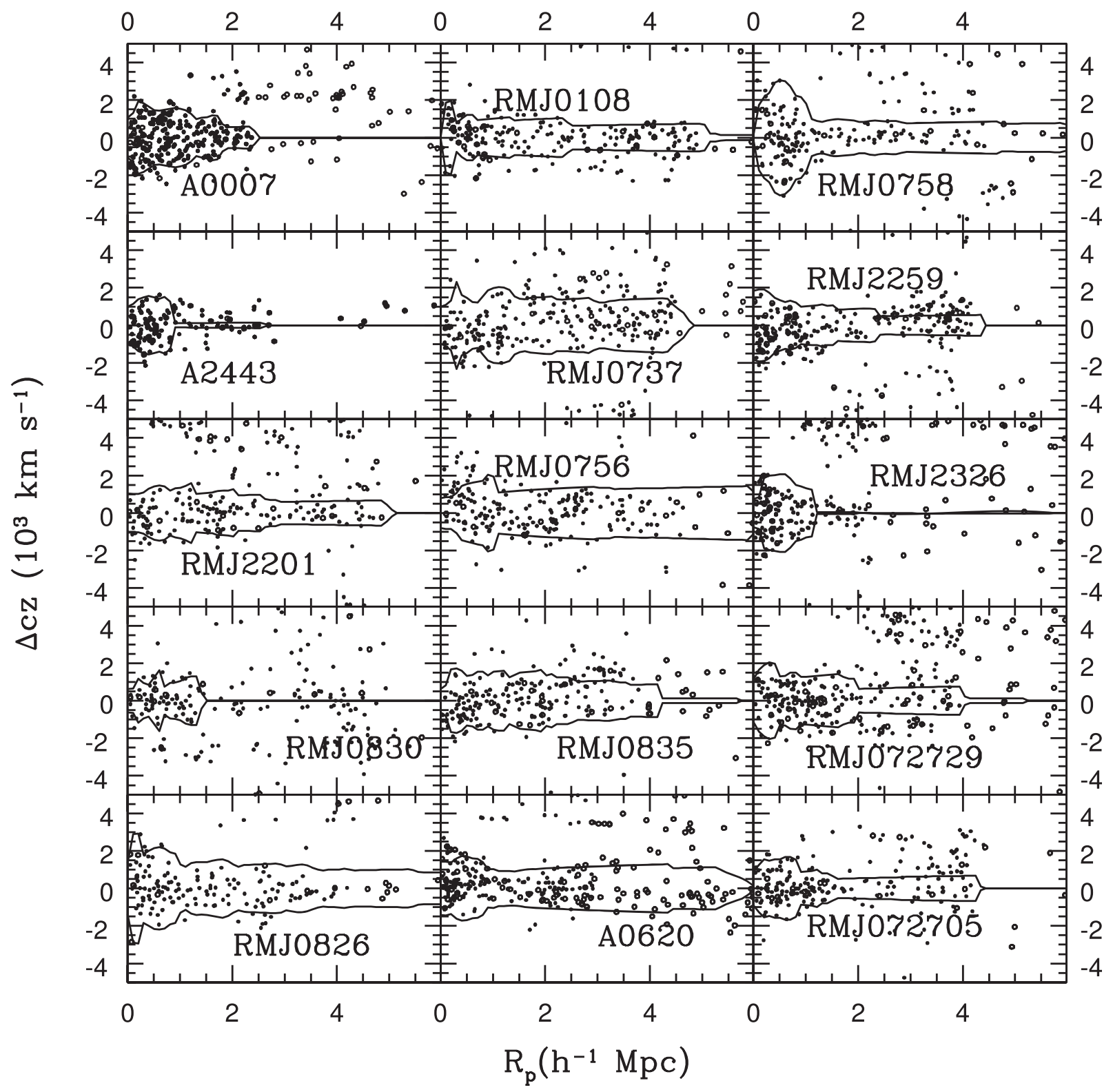

Figure 6. Same as Figure 5.

Figure 6) and is therefore not gravitationally bound. Members of A98SS produce the secondary peak in the redshift histogram of A98S visible in Figure 2.

2. A115. A115 shows two prominent X-ray peaks, termed A115N/A115S, separated by 5!1 or $1 h^{-1} \mathrm{Mpc}$ on the sky (Forman et al. 1981). Chandra observations show that the intracluster medium (ICM) in the cores of A115N and A115S is cooler than the ICM in the outer parts, consistent with a subsonic merger (Gutierrez \& Krawczynski 2005). Barrena et al. (2007a) used a redshift survey to probe the dynamics of the cluster merger. They found that $\mathrm{A} 115 \mathrm{~N}$ and $\mathrm{A} 115 \mathrm{~S}$ are separated by about $2000 \mathrm{~km} \mathrm{~s}^{-1}$ and that the velocity dispersion of A115S is somewhat larger than that of A115N. They also find that a few galaxies are located at lower velocity and centered around a galaxy they term BCM-D. These galaxies are located outside the caustics in Figure 5. We treat A115N and A115S as a combined system.
From the extended sample (HeCS-red-ext), some notable clusters are:

1. A963. A963 was noted by Rozo \& Rykoff (2014) to be an outlier in the Planck-SZ-S/N-richness scaling relation. A963 has the largest X-ray temperature for clusters for its richness. Rozo \& Rykoff (2014) inspected the DR8 photometry around this cluster and concluded that there was a systematic uncertainty in the photometric background surrounding this cluster due to a bright star. The large uncertainties in estimated colors of galaxies may scatter many of them outside of the red sequence. Andreon (2014) estimated the richness of A963 and found a much larger value than the redMaPPer algorithm. Similarly, HeCS (Rines et al. 2013; Hwang et al. 2014) showed that this cluster contains many spectroscopically confirmed members. We show below (Section 4.4) that the richness estimate from our spectroscopy places this 


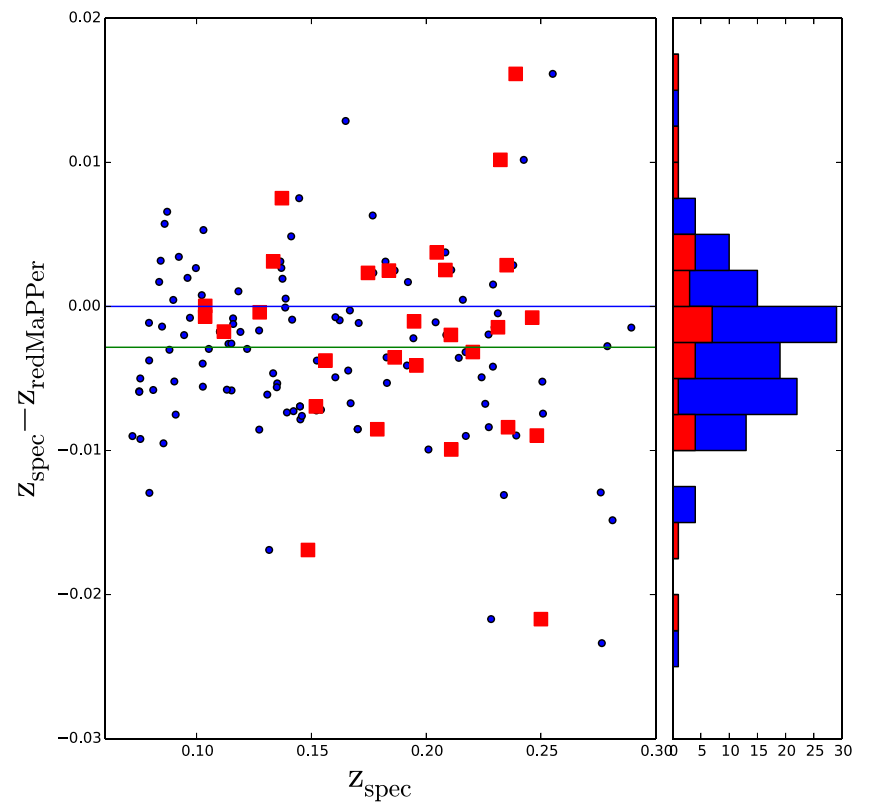

Figure 7. Spectroscopic redshifts vs. photometric redshifts from the redMaPPer catalog. Red squares and blue circles show clusters from HeCS-red and HeCSred-ext respectively. The blue horizontal lines shows zero offset. The green horizontal line shows the mean offset of the HeCS-red-ext sample.

Table 4

Scaling Relations between Dynamical Masses and Richnesses

\begin{tabular}{lccc}
\hline \hline Relation & $b$ & $a$ & $\sigma_{y}$ \\
\hline$P\left(\sigma_{p} \mid \lambda\right)$ & $0.38_{-0.18}^{+0.19}$ & $0.050_{-0.023}^{+0.024}$ & $0.094_{-0.014}^{+0.018}$ \\
Extended sample & $0.240_{-0.046}^{+0.047}$ & $0.046 \pm 0.011$ & $0.090_{-0.007}^{+0.008}$ \\
\hline$P\left(M_{200} \mid \lambda\right)$ & $0.99_{-0.44}^{+0.46}$ & $0.052 \pm 0.059$ & $0.252_{-0.035}^{+0.044}$ \\
Extended sample & $0.58_{-0.14}^{+0.14}$ & $0.005_{-0.032}^{+0.031}$ & $0.276_{-0.018}^{+0.021}$ \\
\hline$P\left(\sigma_{p} \mid N_{\text {gal }}\right)$ & $0.52_{-0.18}^{+0.19}$ & $0.059_{-0.022}^{+0.021}$ & $0.084_{-0.014}^{+0.018}$ \\
\hline
\end{tabular}

Note. Fits are of the relation $P(y \mid x)$, assuming the linear form $\log y=$ $a+b \log x$ with intrinsic scatter $\sigma_{\log y}$ in the relation at fixed values of $\log x$. Richnesses are divided by 100 , velocity dispersions are divided by $700 \mathrm{~km} \mathrm{~s}^{-1}$, and logarithmic masses are subtracted by 14.5 .

cluster much closer to the locus of points for the other clusters.

2. A1068. This cluster has a large velocity dispersion for its richness. A1068 was also noted as an outlier in the massrichness relation by Andreon (2014).

3. A1682. This cluster has a large velocity dispersion for its richness.

4. MS0906 and A750. This pair of nearby clusters was noted in HeCS and found to have a large weak lensing mass for its caustic mass, presumably because the lensing mass includes both clusters while the caustic masses are able to separate the clusters (Geller et al. 2013). Perhaps unsurprisingly, these clusters appear to be conflated by the redMaPPer algorithm as well, making them unusually rich for their velocity dispersions.

Using our spectroscopy to estimate the richnesses of these clusters, both clusters lie much closer to the main locus of points in both scaling relations (Section 4.4). This cluster pair is a good example of the "catastrophic outliers" expected in richness-based cluster catalogs: two clusters of roughly comparable mass and richness that are also closely separated in redshift. Without spectroscopic redshifts, this cluster pair would be counted as a single cluster of roughly twice the mass of the individual clusters, resulting in a biased estimation of the cluster mass function.

5. A1758N and A1758S. Another pair of nearby clusters is A1758N and A1758S, each of which is composed of two merging clusters (David \& Kempner 2004; Okabe \& Umetsu 2008; Ragozzine et al. 2012). The redMaPPer algorithm identifies $\mathrm{A} 1758 \mathrm{~N}$ and $\mathrm{A} 1758 \mathrm{~S}$ as separate systems, but it does not detect their components. Because the velocity distributions of the two clusters comprising A1758N overlap significantly, the measured velocity dispersion is probably not much larger than the velocity dispersions of the individual clusters. Thus, it is not surprising that the redMaPPer richness of the two clusters comprising $\mathrm{A} 1758 \mathrm{~N}$ is large compared to its velocity dispersion.

\section{Discussion}

\subsection{Reliability of Cluster Identification and Photometric Redshifts in the redMaPPer DR8 Catalog}

Our MMT/Hectospec observations of redMaPPer-selected clusters show that, at the relatively high richnesses of our target clusters $(\lambda \geqslant 64)$, cluster candidates in the redMaPPer catalog correspond to real overdensities in redshift space. Further, the redshift of the primary overdensity at the spatial position of the cluster candidate agrees well with the estimated redshift in the redMaPPer catalog.

\subsection{Spectroscopic Completeness}

Figure 12 shows the spectroscopic completeness of the HeCS-red sample measured inside the Abell radius. The upper panel shows the fraction of target galaxies (those within $0.1 \mathrm{mag}$ of the red sequence) with redshifts and the fraction of observed galaxies that are cluster members. These fractions are displayed as functions of fiducial absolute magnitude (that is, the absolute magnitude that confirmed members or unobserved galaxies would have if they were cluster members). For conversion to absolute magnitudes, we use the fitting functions from Westra et al. (2010) based on empirical K-corrections from spectrophotometry with Hectospec and SDSS photometry.

The lower panel of Figure 12 shows the number of spectroscopically confirmed members, confirmed background/foreground galaxies, and unobserved galaxies as a function of fiducial absolute magnitude. Vertical dotted lines indicate absolute magnitudes of $M^{*}, M^{*}+1$, and $M^{*}+1.75$. We categorize galaxies in these luminosity bins as bright, intermediate, and faint, respectively. The membership fraction of faint galaxies is similar to the membership fraction of bright galaxies. The spectroscopic completeness decreases steadily with decreasing luminosity, but the completeness fraction of faint galaxies $(\sim 40 \%)$ is sufficient to enable completeness corrections for the red-sequence luminosity function and cluster richness. The dashed line in the lower panel of Figure 12 shows the total number of members in all HeCS-red clusters after correcting for incompleteness. 

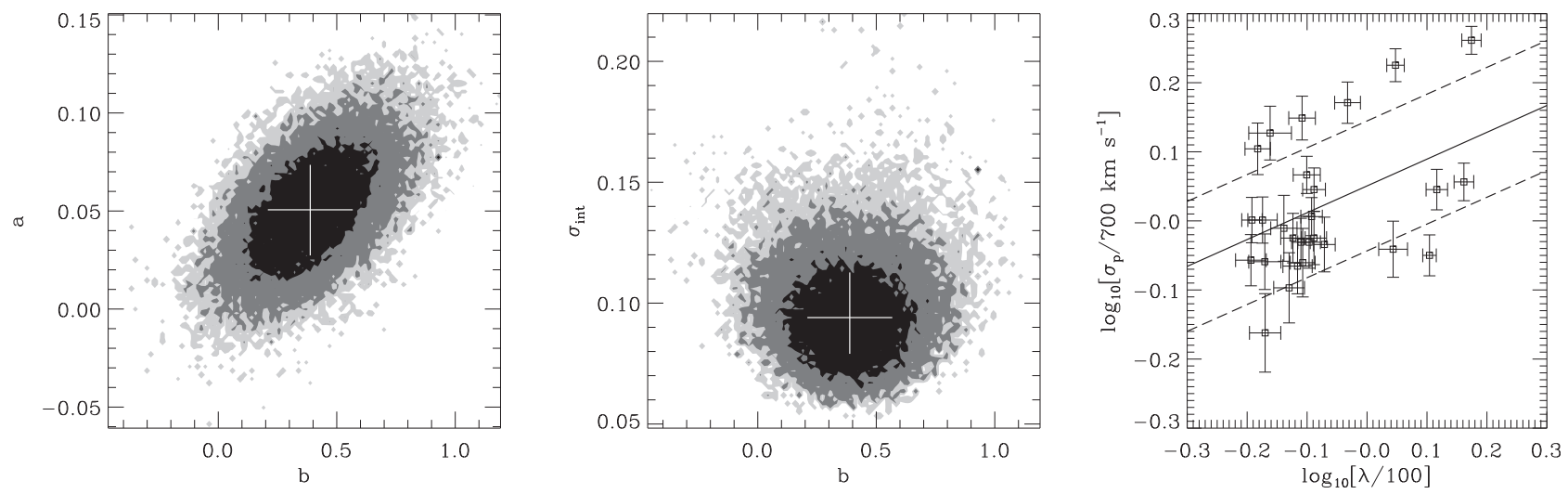

Figure 8. Left panel: marginalized probability distribution function of the intercept and slope of the relation $P\left(\sigma_{p} \mid \lambda\right)=a+b \log (\lambda)$ from MCMC analysis. The shaded areas with decreasing darkness show the $68.3 \%, 95.4 \%$, and $99.7 \%$ marginalized credible intervals. The white cross shows the $68.3 \%$ marginalized credible interval of each parameter. Middle panel: similar to the left panel for the intrinsic scatter $\sigma_{\text {int }}$ and intercept $b$ of the scaling relation. Right panel: scaling relation between projected velocity dispersion $\sigma_{p}$ and the richness proxy $\lambda$ for clusters in the redMaPPer-complete sample (HeCS-red). Dashed lines indicate the intrinsic scatter for individual clusters.

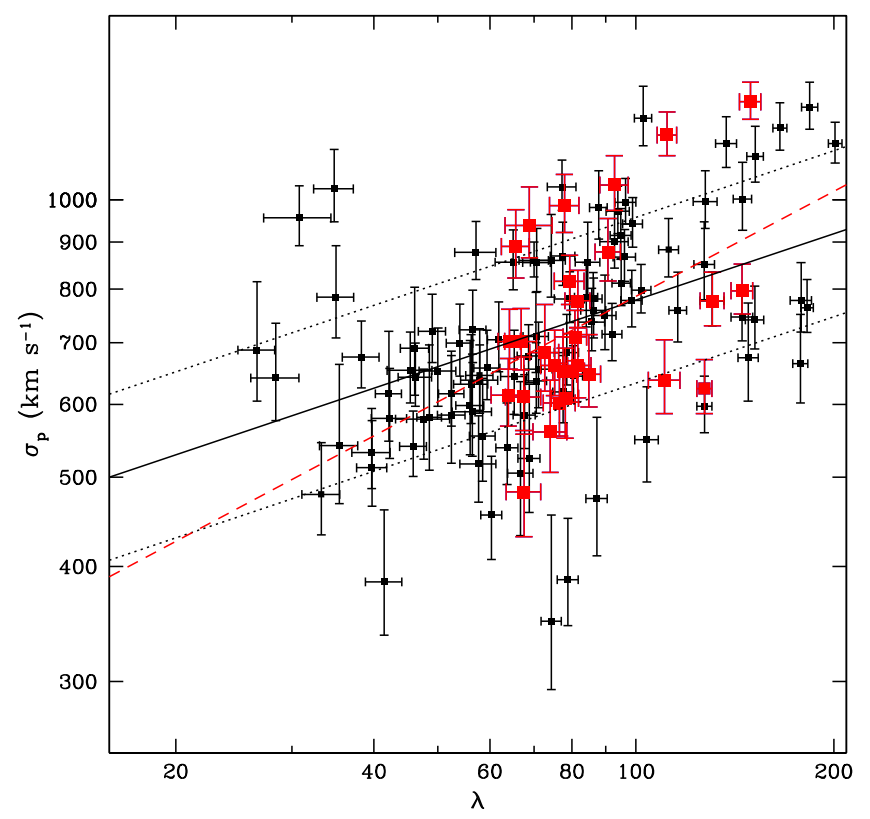

Figure 9. Scaling relation between projected velocity dispersion $\sigma_{p}$ and the richness proxy $\lambda$ for clusters in the redMaPPer-complete sample supplemented with clusters from other surveys (Sample 2). Red and black points show clusters from HeCS-red and HeCS-red-ext, respectively. The thick solid line shows the best-fit relation of $P\left(\sigma_{p} \mid \lambda\right)$ for the HeCS-red-ext sample with the intrinsic scatter shown as dotted lines. For reference, the dashed line shows the best-fit relation for the HeCS-red sample.

\subsection{Evaluation of Photometric Redshift Membership Probability}

Along with the cluster catalog, Rykoff et al. (2014) released a catalog of candidate cluster members where each galaxy has an assigned probability $P_{\text {mem }}$ that it is a member of a cluster. Here, we use our spectroscopic redshifts to assess the reliability of these probability estimates.

We match our spectroscopic catalog to the redMapper membership catalog. We identify 2159 galaxies in common, of which 1710 are spectroscopically confirmed members (i.e., lie within the caustics). Figure 13 shows the spectroscopic membership fraction $f_{\text {mem,spec }}=N_{\mathrm{RMmem}, \text { spec }} / N_{\mathrm{RMmem}}$ as a function of $P_{\mathrm{mem}}$. $N_{\mathrm{RMmem}}$ is the number of redMaPPer candidate members with spectroscopic redshifts and

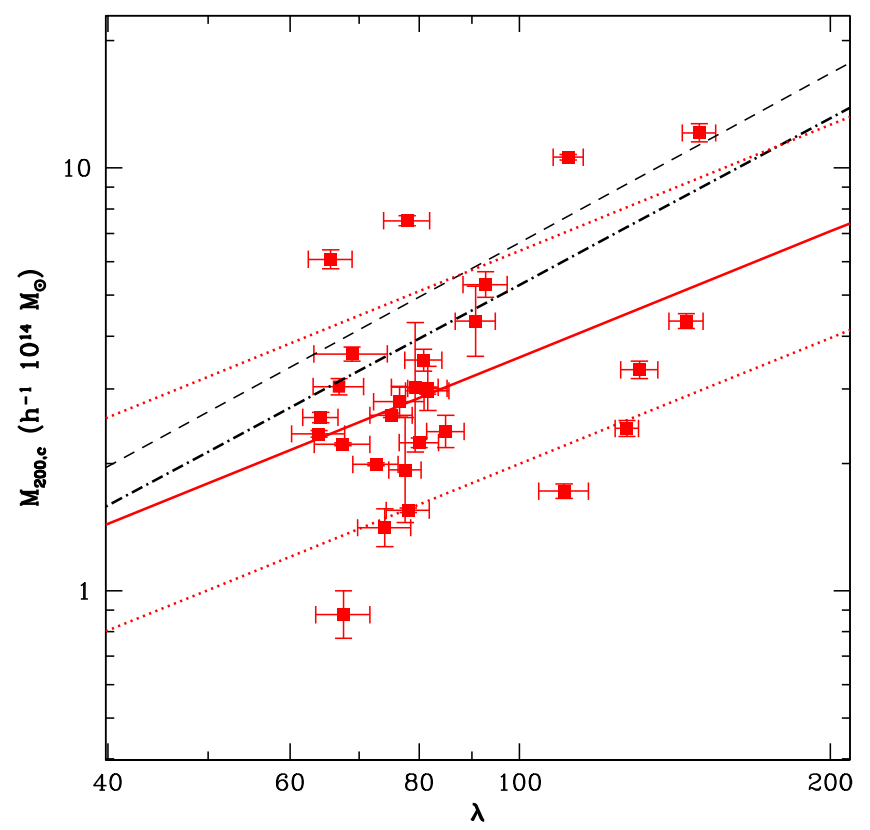

Figure 10. Similar to Figure 8, but for the scaling relation between caustic mass $M_{200}$ and richness proxy $\lambda$ for the HeCS-red sample. The red solid and dotted lines show the best-fit relation for HeCS-red-ext and the intrinsic scatter for individual clusters. The black dashed-dotted line shows the mass- $\lambda$ scaling relation from a kinematic analysis of redMapper clusters using sparsely sampled spectroscopy (Farahi et al. 2016). The dashed line shows the mass- $\lambda$ scaling relation from a weak lensing analysis of stacked redMapper clusters (Simet et al. 2017).

$N_{\text {RMmem,spec }}$ is the number of redMaPPer candidate members classified as members with the caustic technique. The member fraction is larger than $P_{\text {mem }}$ for all but the largest values of $P_{\text {mem }}$. The dashed line in Figure 13 shows an ordinary least squares fit to the data. The equation for the line is $f_{\text {mem,spec }}=(0.361 \pm 0.097) P_{\mathrm{mem}}+(0.496 \pm 0.056)$, indicating that $P_{\text {mem }}$ underestimates the actual membership probability at $P_{\text {mem }}<0.8$ and overestimates the membership probability at $P_{\text {mem }}>0.8$.

Our results are not consistent with Rozo et al. (2015), who conclude from a comparison of redMaPPer cluster candidates and GAMA redshifts that redMaPPer probabilities are largely accurate with some small systematic effects. Their results are 


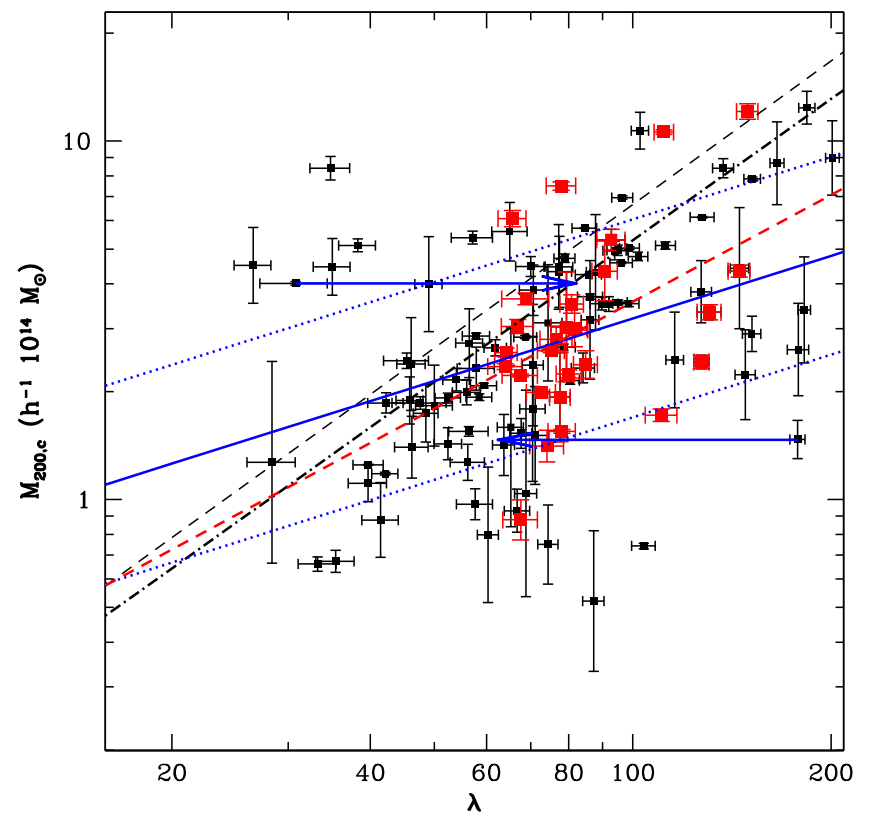

Figure 11. Similar to Figure 9 , but for the scaling relation between caustic mass $M_{200}$ and richness proxy $\lambda$. Red and black points are clusters from HeCSred and HeCS-red-ext respectively. The blue solid and dotted lines show the best-fit relation for HeCS-red-ext and the intrinsic scatter for individual clusters. For comparison, the red dashed line shows the best-fit relation for the HeCS-red sample. The black dashed-dotted line and dashed line show the mass- $\lambda$ scaling relations from Farahi et al. (2016) and Simet et al. (2017), respectively. Arrows show spectroscopic richness estimates for A0963 and MS0906. The richness of A0963 was underestimated due to problems with photometry in the redMaPPer analysis and the richness of MS0906 is overestimated due to a superposition with A0750.

based on clusters with smaller richnesses $(\lambda<64)$ than the HeCS-red clusters. Sohn et al. (2018) perform a similar comparison using redshifts from the HectoMAP redshift survey (Geller et al. 2014; Hwang et al. 2016). The redMaPPer clusters in HectoMAP have smaller richnesses than HeCS-red clusters, and the membership fraction they measure (dotted line in Figure 13) is consistent with Rozo et al. (2015). Thus, the discrepant results on membership probabilities are consistent with a richness effect such that values of $P_{\text {mem }}$ of $0.05-0.7$ from the redMaPPer algorithm underestimate the true membership probability for galaxies in high-richness clusters.

Figure 13 shows a color-magnitude diagram for redMaPPer candidate members from the HeCS-red clusters. For this figure, we plot fiducial colors and fiducial absolute magnitudes as if these galaxies were at the redshift of the cluster. Candidates with $P_{\text {mem }}<0.4$ typically lie further away from the red sequence than candidates with larger $P_{\text {mem }}$. Thus, the high spectroscopic membership fraction for galaxies with small $P_{\text {mem }}$ can largely be attributed to spectroscopic members that are somewhat faint and somewhat bluer than the red sequence.

\subsection{Richness Estimated via Spectroscopy}

Our spectroscopic data enable a test of the photometric richness parameter $\lambda$. The richness parameter $\lambda$ is the estimated number of galaxies projected inside a cutoff radius that scales with the parameter $\lambda$. That is, the cutoff radius is larger for clusters with larger $\lambda$.

We adopt a simplified approach to estimate the richness of clusters using our spectroscopy. We use a fixed cutoff radius of

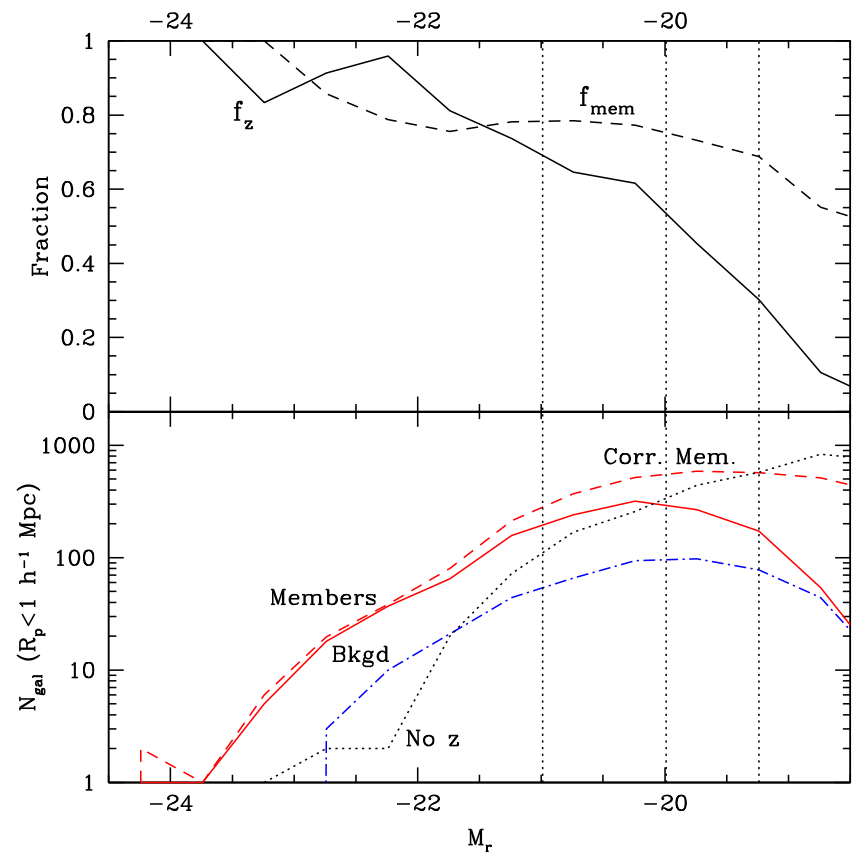

Figure 12. Top panel: a fraction of candidate red-sequence galaxies (projected radius within $1.0 \mathrm{Mpc}$ and color within $0.1 \mathrm{mag}$ of the red sequence) with spectroscopic redshifts as a function of fiducial absolute magnitude (i.e., nonmembers and unobserved galaxies are treated as if located at the distance of the cluster). The dashed line shows the membership fraction of spectroscopically observed galaxies. Vertical dotted lines (from left to right) indicate absolute magnitudes of $M^{*}, M^{*}+1$, and $M^{*}+1.75$. Bottom panel: the number of candidate red-sequence galaxies vs. fiducial absolute magnitude. Red solid, blue dashed-dotted, and black dotted lines, respectively, show the number of spectroscopically confirmed members, spectroscopically confirmed background (and foreground) galaxies, and galaxies without redshifts. The dashed line show the number of members after correcting for incompleteness.

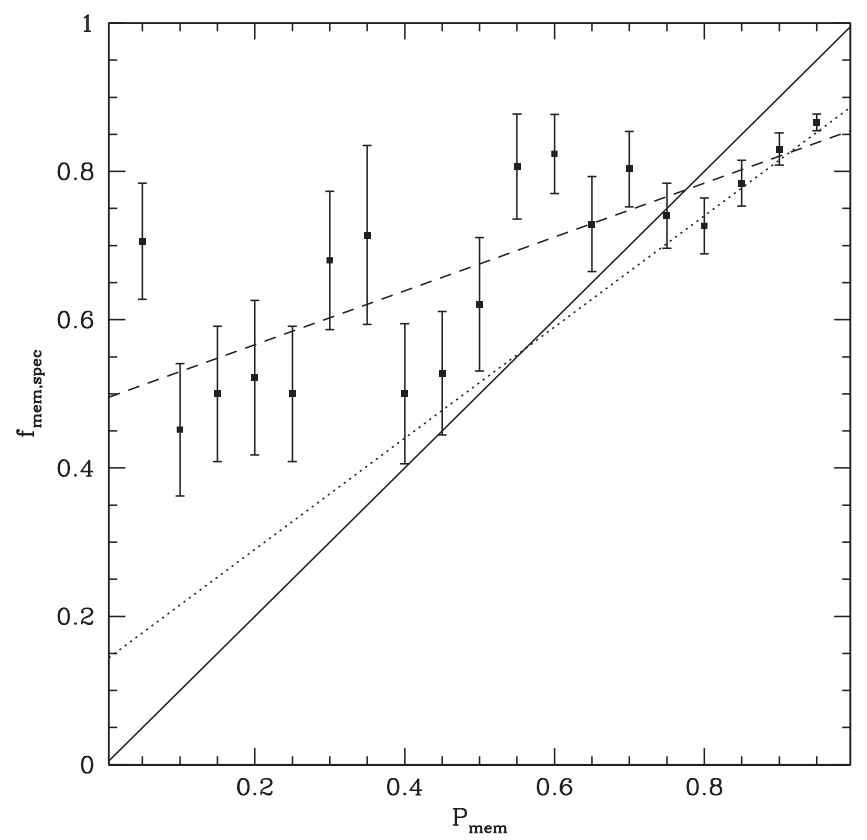

Figure 13. Spectroscopic member fractions of redMaPPer candidate members in bins of membership probability $P_{\mathrm{mem}}$. The solid line shows $f_{\mathrm{mem}, \mathrm{spec}}=$ $P_{\text {mem }}$ and the dashed line shows an ordinary least squares fit to the data. Uncertainties are computed assuming a binomial distribution. The dotted line shows a similar comparison from analysis of (mostly smaller richness) redMaPPer clusters in the HectoMAP redshift survey (Sohn et al 2018). 


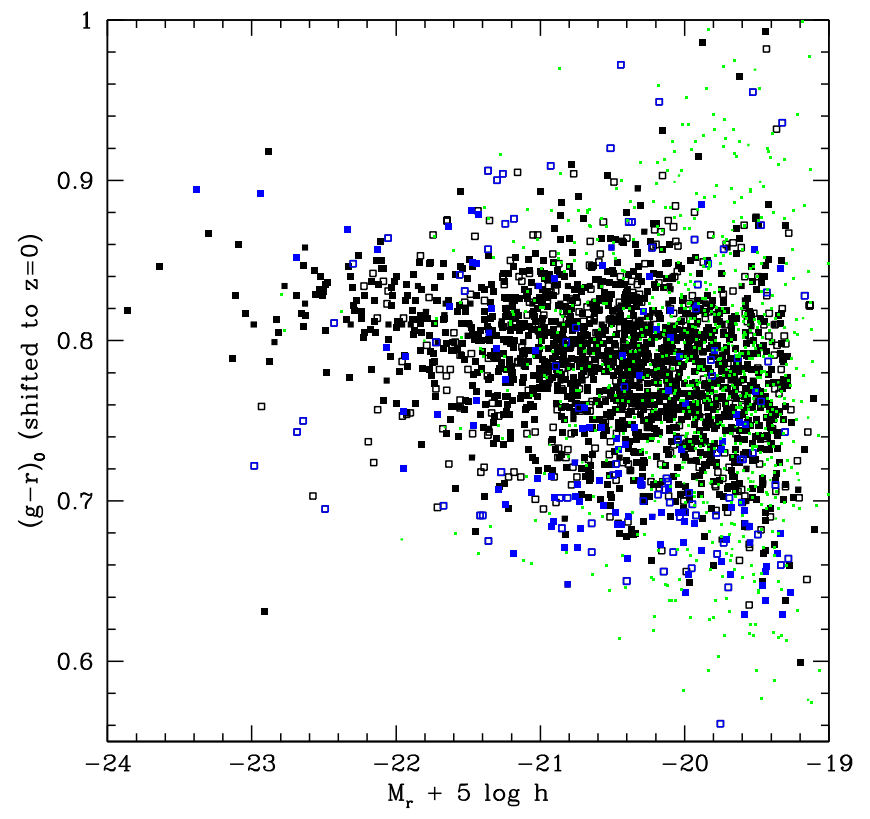

Figure 14. Fiducial color-magnitude diagram for redMaPPer candidate members. Absolute magnitudes and colors are corrected to $z=0$ assuming that all galaxies lie at the redshift of the target cluster. Filled (open) points show spectroscopically identified members (non-members). Blue points indicate galaxies with membership probability $P_{\text {mem }}<0.4$. Small green points show redMaPPer candidate members without spectroscopic redshifts.

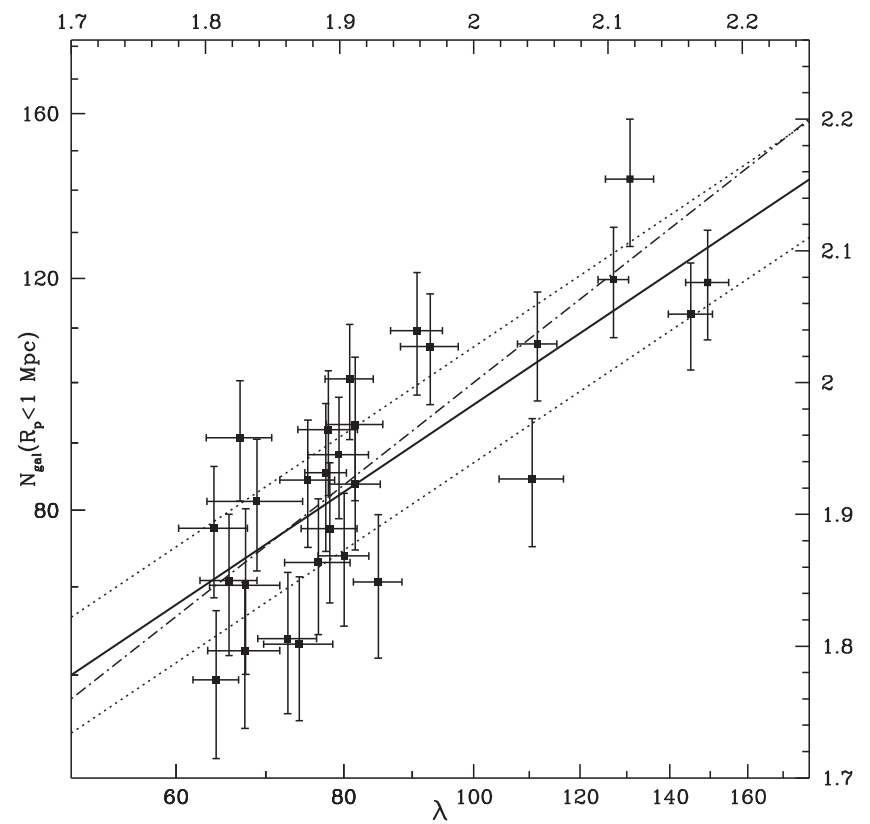

Figure 15. Richness parameter $\lambda$ vs. estimated red-sequence richness within $1 h^{-1} \mathrm{Mpc}$ as estimated from spectroscopically classified members. The dasheddotted line shows a power law with slope 0.8 , corresponding to the slope expected if correcting the richness parameter $\lambda$ to a fixed aperture of $1 h^{-1} \mathrm{Mpc}$ with a richness within $R_{\text {ap }}$ proportional to $R_{\text {ap }}$. The thick solid line shows the best-fit relation of $P\left(N_{\mathrm{gal}} \mid \lambda\right)$ with the intrinsic scatter shown as dotted lines.

$1 h^{-1} \mathrm{Mpc}$ for all clusters (this is the cutoff radius redMaPPer adopts for richness parameter $\lambda=100$ ). We only include galaxies on the photometric red sequence. We then measure the membership fraction in three bins of absolute magnitude M: brighter than $M^{*}, M^{*}<M<M^{*}+1$, and $M^{*}+1<M<$ $M^{*}+1.75$. Figure 12 shows that the HeCS-red spectra are

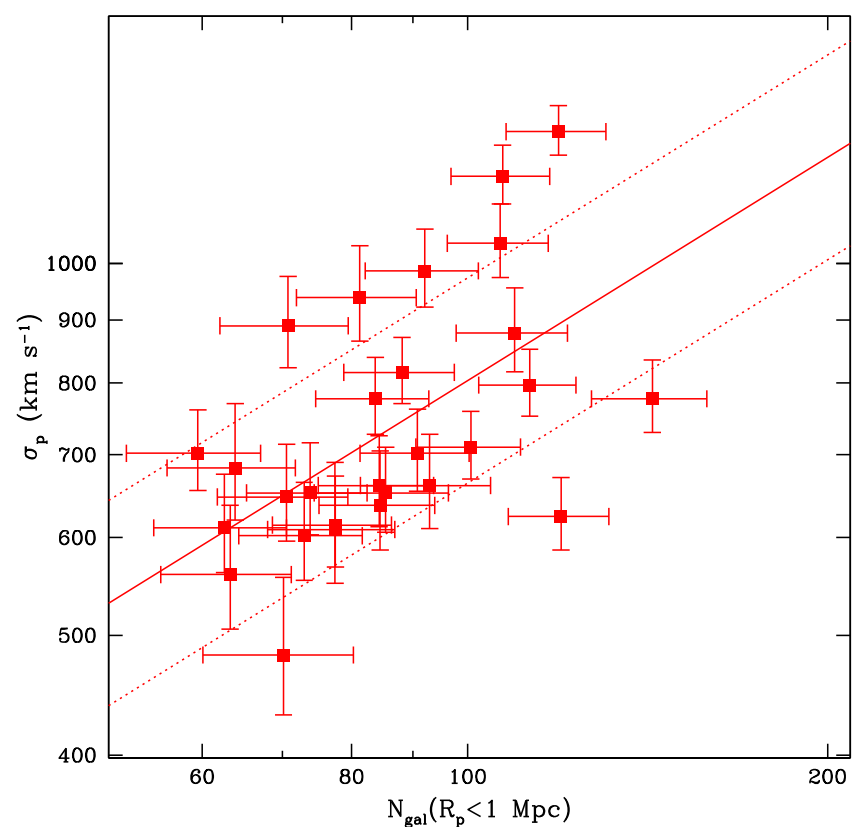

Figure 16. Projected velocity dispersion $\sigma_{p}$ versus estimated red-sequence richness within $1 \mathrm{~h}^{-1} \mathrm{Mpc}$ as estimated from spectroscopically classified members. The Pearson correlation coefficient $(0.535)$ is larger than for the $\sigma_{p}-\lambda$ relation (0.426; right panel of Figure 8), suggesting that the spectroscopic richness estimates are more tightly correlated with cluster mass than photometric richness estimates.

$\sim 80 \%$ complete in the brightest bin, $\sim 60 \%$ complete in the intermediate bin, and $\sim 40 \%$ complete in the faintest bin. We correct for the incompleteness by dividing the number of members in each absolute magnitude bin by the completeness fraction of that bin. We then sum the corrected counts to find the total number of cluster galaxies $N_{\mathrm{gal}}$ within $1 h^{-1} \mathrm{Mpc}$ in projection. We apply the same procedure to estimate the richnesses of two outliers in the HeCS-red-ext sample, A0963 and MS0906. These clusters are no longer outliers in the $M_{200}-\lambda$ relation when we use our spectroscopic richness estimates (Figure 11).

Figure 14 compares our spectroscopic richness estimates with the photometric redMaPPer richness estimates. The two estimates of richness agree with each other, suggesting that the photometric richness estimates from redMaPPer are closely related to each other. However, there is a large range in $N_{\text {gal }}$ (up to a factor of two) at fixed $\lambda$. Using the same Bayesian framework as above, the best-fit relation is $\log \left(N_{\text {gal }} / 100\right)=$ $(0.68 \pm 0.12) \log (\lambda / 100)-(0.017 \pm 0.015) \quad$ with intrinsic scatter $\sigma_{\text {int }}=0.044_{-0.013}^{+0.015}$. The solid line in Figure 14 shows this relation.

One subtle aspect of the richness comparison is that the redMaPPer algorithm does not use a fixed radial aperture to measure richness. Instead, it uses a radial aperture that scales with the estimated richness; in practice, the aperture and richness parameters are fit jointly. The redMaPPer radial aperture is given by $R_{\mathrm{ap}, \mathrm{RM}}=(\lambda / 100)^{0.2}$. For our redMaPPerselected sample, $R_{\mathrm{ap}, \mathrm{RM}}$ changes by $18.5 \%$ across the range of $\lambda$ covered by the sample. We estimate the expected number of members within a fixed radial aperture of $1 h^{-1} \mathrm{Mpc}$ by assuming that the number of members increases approximately linearly with radius (consistent with an NFW number density profile; see also Figure 16 of Rykoff et al. 2014). Under this assumption, the number of galaxies $\lambda_{1 \mathrm{Mpc}}$ expected within a 
fixed radial aperture of $1 h^{-1} \mathrm{Mpc}$ would be about $10 \%$ larger (smaller) than $\lambda$ for clusters with the smallest (largest) $\lambda$ in our sample. The dashed-dotted line in Figure 14 shows this prediction. The agreement between the predicted relation and our measured relation shows that the average richness of redMaPPer clusters at fixed $\lambda$ is approximately equal to the richness $N_{\text {gal }}$ measured with spectroscopy. However, the large range of $N_{\text {gal }}$ at fixed $\lambda$ indicates that there is significant uncertainty in this estimate for individual clusters.

Finally, Figure 16 shows projected velocity dispersion $\sigma_{p}$ versus the spectroscopic richness estimates. The Pearson correlation coefficient $(0.535)$ is larger than the correlation coefficient of the $\sigma_{p}-\lambda$ relation (0.426), suggesting that the spectroscopic richness estimates are more tightly correlated with cluster mass than photometric richness estimates. Table 4 lists the best-fit parameters of the $\sigma_{p}-N_{\text {gal }}$ relation. The intrinsic scatter is slightly smaller than for the $\sigma_{p}-\lambda$ relation.

\section{Conclusions}

We used spectroscopy from MMT/Hectospec to test the redMaPPer algorithm for detecting clusters and estimating richnesses. Our test is limited to high-richness, low-redshift systems where we can measure large numbers of redshifts with MMT/Hectospec. We construct two samples, one selected purely on richness (HeCS-red), and a larger, sample that includes clusters selected on X-ray flux or SZ signal (HeCS-red-ext).

We confirm that all of the cluster candidates in this highrichness sample are genuine clusters, although several systems show evidence of multiple structures along the line of sight. The extended HeCS-red-ext sample shows evidence for bias in photometric redshifts of redMaPPer clusters, with $z_{\text {spec }}-z_{\mathrm{RM}}=$ $-0.0028 \pm 0.0005$. This bias may be an effect of including background galaxies as cluster members when estimating photometric redshifts.

For the richness-selected HeCS-red sample, measured velocity dispersions correlate with cluster richness, and the scaling relation contains $24 \%$ intrinsic scatter in velocity dispersion at fixed richness. Four clusters with small velocity dispersion given their estimated richness have background redshift peaks of nearby but unbound structure. Nearby but unbound structure may produce overestimates of richness from photometric data. The enlarged sample shows larger scatter and some outliers. Inspection of some of these outliers reveals that they can be caused both by problematic photometry (A963) and by "catastrophic" superpositions of nearly equal-mass clusters (MS0906/A750). The range of velocity dispersion at fixed richness estimate $\lambda$ is a factor of two (three) for the HeCS-red (HeCS-red-ext) sample, and the range of measured $M_{200}$ at fixed $\lambda$ is a factor of roughly 10 (20). Thus, the richness estimate $\lambda$ is a low-precision predictor of $\sigma_{p}$ or $M_{200}$ for individual clusters.

We compare spectroscopic membership classification to photometric membership classification. On average, the membership probability (estimated from photometry) of spectroscopically confirmed non-members is smaller than the membership probability of spectroscopically confirmed members. For the high-richness, low-redshift clusters in HeCS-red, the membership probabilities in redMaPPer appear to be underestimated for low-probability galaxies $\left(P_{\mathrm{mem}}<0.75\right)$ and overestimated for high-probability galaxies $\left(P_{\mathrm{mem}} \geqslant 0.8\right)$.

We estimate cluster richnesses from our spectroscopically determined member catalogs. The spectroscopic richnesses correlate well with the photometric richness estimates from redMaPPer. Apparently, the underestimated probabilities for low- $P_{\text {mem }}$ candidate members are roughly balanced by the overestimated probabilities for high- $P_{\text {mem }}$ candidate members. However, there is substantial scatter in the relation; the range of spectroscopic richness at fixed photometric richness is nearly a factor of two.

The spectroscopic richness estimates are more tightly correlated with the measured velocity dispersion than the photometric richness estimates. Although observationally expensive, the spectroscopic redshifts improve the quality of richness as a cluster mass proxy.

Overall, our spectroscopic survey of red-sequence-selected clusters suggests that these cluster candidates are associated with significant overdensities in redshift space. Photometricbased estimates of redshift, richness, and membership probability correlate well with spectroscopic measures, although the scatter in individual objects is substantial. Larger samples of red-sequence-selected clusters are necessary to provide robust constraints on the scaling relations between photometric richness and dynamical mass proxies like velocity dispersion.

HeCS-red focuses on low-redshift, high-richness clusters from the redMaPPer catalog. RedMaPPer clusters cover the range $0.08<z<0.6$ and richnesses $\lambda>20$. Thus, HeCS-red and HeCS-red-ext provide only a partial picture of the relationship between the spectroscopic properties of clusters and their redMaPPer counterparts. In a complementary paper, Sohn et al. (2018) test the full redshift and richness range of the redMaPPer catalog.

K.R. thanks the University of Washington for hosting a visit during which some of the work was performed. M.J.G. is supported by the Smithsonian Institution. A.D. acknowledges support from the INFN grant InDark. J.S. acknowledges the support of a CfA Fellowship. We thank Susan Tokarz for reducing the spectroscopic data, and Perry Berlind and Mike Calkins for assisting with the observations. We also thank the telescope operators at the MMT and Nelson Caldwell for scheduling Hectospec queue observations. We thank the Telescope Data Center for assistance with data reduction.

Funding for the Sloan Digital Sky Survey (SDSS) has been provided by the Alfred P. Sloan Foundation, the Participating Institutions, the National Aeronautics and Space Administration, the National Science Foundation, the U.S. Department of Energy, the Japanese Monbukagakusho, and the Max Planck Society. The SDSS website is http://www.sdss.org/. The SDSS is managed by the Astrophysical Research Consortium (ARC) for the Participating Institutions. The Participating Institutions are The University of Chicago, Fermilab, the Institute for Advanced Study, the Japan Participation Group, The Johns Hopkins University, the Korean Scientist Group, Los Alamos National Laboratory, the Max-Planck-Institute for Astronomy (MPIA), the Max-Planck-Institute for Astrophysics (MPA), New Mexico State University, University of Pittsburgh, University of Portsmouth, Princeton University, the United States Naval Observatory, and the University of Washington.

Facilities: MMT (Hectospec), FLWO: 1.5 m (FAST).

\section{ORCID iDs}

Margaret J. Geller (1) https://orcid.org/0000-0002-9146-4876 Ho Seong Hwang (1) https://orcid.org/0000-0003-3428-7612 Jubee Sohn ำ https://orcid.org/0000-0002-9254-144X 


\section{References}

Abraham, R. G., Smecker-Hane, T. A., Hutchings, J. B., et al. 1996, ApJ, 471,694

Adelman-McCarthy, J. K., Agüeros, M. A., Allam, S. S., et al. 2006, ApJS, 162,38

Ahn, C. P., Alexandroff, R., Allende Prieto, C., et al. 2014, ApJS, 211, 17

Aihara, H., Allende Prieto, C., An, D., et al. 2011, ApJS, 193, 29

Albareti, F. D., Allende Prieto, C., Almeida, A., et al. 2017, ApJS, 233, 25A

Andreon, S. 2014, A\&A, 570, L10

Barrena, R., Boschin, W., Girardi, M., \& Spolaor, M. 2007a, A\&A, 467, 37

Barrena, R., Boschin, W., Girardi, M., \& Spolaor, M. 2007b, A\&A, 469, 861

Beers, T. C., Geller, M. J., \& Huchra, J. P. 1982, ApJ, 257, 23

Benson, B. A., de Haan, T., Dudley, J. P., et al. 2013, ApJ, 763, 147

Biviano, A., \& Girardi, M. 2003, ApJ, 585, 205

Biviano, A., Murante, G., Borgani, S., et al. 2006, A\&A, 456, 23

Böhringer, H., Schuecker, P., Guzzo, L., et al. 2004, A\&A, 425, 367

Buchner, J., \& Gruberbauer, M. 2011, APEMoST (Automated Parameter Estimation and Model Selection Toolkit), http://apemost.sourceforge.net/

Danese, L., de Zotti, G., \& di Tullio, G. 1980, A\&A, 82, 322

David, L. P., \& Kempner, J. 2004, ApJ, 613, 831

de Haan, T., Benson, B. A., Bleem, L. E., et al. 2016, ApJ, 832, 95

Diaferio, A. 1999, MNRAS, 309, 610

Diaferio, A. 2009, arXiv:0901.0868

Diaferio, A., \& Geller, M. J. 1997, ApJ, 481, 633

Diaferio, A., Geller, M. J., \& Rines, K. J. 2005, ApJL, 628, L97

Evrard, A. E., Bialek, J., Busha, M., et al. 2008, ApJ, 672, 122

Fabricant, D., Cheimets, P., Caldwell, N., \& Geary, J. 1998, PASP, 110, 79

Fabricant, D., Chilingarian, I., Hwang, H. S., et al. 2005, PASP, 117, 1411

Faltenbacher, A., \& Diemand, J. 2006, MNRAS, 369, 1698

Farahi, A., Evrard, A. E., Rozo, E., Rykoff, E. S., \& Wechsler, R. H. 2016, MNRAS, 460, 3900

Forman, W., Bechtold, J., Blair, W., et al. 1981, ApJL, 243, L133

Geller, M. J., Diaferio, A., Rines, K. J., \& Serra, A. L. 2013, ApJ, 764, 58

Geller, M. J., Hwang, H. S., Diaferio, A., et al. 2014, ApJ, 783, 52

Gifford, D., \& Miller, C. J. 2013, ApJL, 768, L32

Gladders, M. D., \& Yee, H. K. C. 2000, AJ, 120, 2148

Gladders, M. D., \& Yee, H. K. C. 2005, ApJS, 157, 1

Gruberbauer, M., Kallinger, T., Weiss, W. W., \& Guenther, D. B. 2009, A\&A, 506, 1043

Gutierrez, K., \& Krawczynski, H. 2005, ApJ, 619, 161

Hasselfield, M., Hilton, M., Marriage, T. A., et al. 2013, JCAP, 7, 8

Henry, J. P., Evrard, A. E., Hoekstra, H., Babul, A., \& Mahdavi, A. 2009, ApJ, 691, 1307

Hwang, H. S., Geller, M. J., Diaferio, A., Rines, K. J., \& Zahid, H. J. 2014, ApJ, 797, 106

Hwang, H. S., Geller, M. J., Park, C., et al. 2016, ApJ, 818, 173
Jones, C., \& Forman, W. 1999, ApJ, 511, 65

Knebe, A., Stoppacher, D., Prada, F., et al. 2018, MNRAS, 474, 5206

Koester, B. P., McKay, T. A., Annis, J., et al. 2007, ApJ, 660, 221

Kurtz, M. J., \& Mink, D. J. 1998, PASP, 110, 934

Lau, E. T., Nagai, D., \& Kravtsov, A. V. 2010, ApJ, 708, 1419

Lauer, T. R., Postman, M., Strauss, M. A., Graves, G. J., \& Chisari, N. E. 2014, ApJ, 797, 82

Mamon, G. A., Biviano, A., \& Boué, G. 2013, MNRAS, 429, 3079

Mantz, A., Allen, S. W., Rapetti, D., \& Ebeling, H. 2010, MNRAS, 406, 1759

Mantz, A. B., von der Linden, A., Allen, S. W., et al. 2015, MNRAS, 446, 2205

Maughan, B. J., Giles, P. A., Rines, K. J., et al. 2016, MNRAS, 461, 4182

Okabe, N., \& Umetsu, K. 2008, PASJ, 60, 345

Old, L., Skibba, R. A., Pearce, F. R., et al. 2014, MNRAS, 441, 1513

Paterno-Mahler, R., Randall, S. W., Bulbul, E., et al. 2014, ApJ, 791, 104

Planck Collaboration et al. 2014a, A\&A, 571, A20

Planck Collaboration et al. 2014b, A\&A, 571, A29

Planck Collaboration, Ade, P. A. R., Aghanim, N., et al. 2016, A\&A, 594, 24

Ragozzine, B., Clowe, D., Markevitch, M., Gonzalez, A. H., \& Bradač, M. 2012, ApJ, 744, 94

Rines, K., \& Diaferio, A. 2006, AJ, 132, 1275

Rines, K., Diaferio, A., \& Natarajan, P. 2007, ApJ, 657, 183

Rines, K., Diaferio, A., \& Natarajan, P. 2008, ApJL, 679, L1

Rines, K., Geller, M. J., Diaferio, A., \& Kurtz, M. J. 2013, ApJ, 767, 15

Rines, K., Geller, M. J., Kurtz, M. J., \& Diaferio, A. 2003, AJ, 126, 2152

Rines, K. J., Geller, M. J., Diaferio, A., \& Hwang, H. S. 2016, ApJ, 819, 63

Rozo, E., \& Rykoff, E. S. 2014, ApJ, 783, 80

Rozo, E., Rykoff, E. S., Becker, M., Reddick, R. M., \& Wechsler, R. H. 2015, MNRAS, 453, 38

Rozo, E., Wechsler, R. H., Rykoff, E. S., et al. 2010, ApJ, 708, 645

Rykoff, E. S., McKay, T. A., Becker, M., et al. 2008, ApJ, 675, 1106

Rykoff, E. S., Rozo, E., Busha, M. T., et al. 2014, ApJ, 785, 104

Sadibekova, T., Pierre, M., Clerc, N., et al. 2014, A\&A, 571, A87

Serra, A. L., \& Diaferio, A. 2013, ApJ, 768, 116

Serra, A. L., Diaferio, A., Murante, G., \& Borgani, S. 2011, MNRAS, 412 800

Simet, M., McClintock, T., Mandelbaum, R., et al. 2017, MNRAS, 466, 3103

Sohn, J., Geller, M. J., Rines, K. J., et al. 2018, ApJ, 856, 172

Vikhlinin, A., Kravtsov, A. V., Burenin, R. A., et al. 2009, ApJ, 692, 1060

Westra, E., Geller, M. J., Kurtz, M. J., Fabricant, D. G., \& Dell'Antonio, I. 2010, PASP, 122, 1258

Wu, H.-Y., Hahn, O., Evrard, A. E., Wechsler, R. H., \& Dolag, K. 2013, MNRAS, 436, 460

Zwicky, F. 1933, AcHPh, 6, 110

Zwicky, F. 1937, ApJ, 86, 217 